\title{
Spectral sequences associated to deformations
}

\author{
Estanislao Herscovich ${ }^{1,2,3}$
}

Received: 1 September 2015 / Accepted: 27 May 2016 / Published online: 13 June 2016

(C) Tbilisi Centre for Mathematical Sciences 2016

\begin{abstract}
Lapin has constructed a multiplicative spectral sequence from a deformation of an $A_{\infty}$-algebra. In particular, as noted by the same author, one can apply this construction to a deformation induced by a filtration of an $A_{\infty}$-algebra. A question that naturally appears is whether this latter multiplicative spectral sequence is isomorphic to the one that is canonically associated to the filtration and that typically appears in basic textbooks on homological algebra. We provide a positive answer to the previous question and we also explain the interesting constructions of Lapin in more structural terms.
\end{abstract}

Keywords Homological algebra $\cdot$ Spectral sequences $\cdot$ Dg algebras $\cdot A_{\infty}$-algebras

Mathematics Subject Classification 14D15 - 16E45 - 16S80 - 16W70 - 18G40 • $18 \mathrm{G} 55 \cdot 55 \mathrm{~T} 05$

Communicated by Hvedri Inassaridze.

The author would also like to thank the Alexander von Humboldt Foundation and the Bielefeld University, for their support during part of this work. This work was also partially supported by UBACYT 20020130200169BA, UBACYT 20020130100533BA, PIP-CONICET 2012-2014 11220110100870 , MathAmSud-GR2HOPF, PICT 2011-1510 and PICT 2012-1186.

$\bowtie \quad$ Estanislao Herscovich

Estanislao.Herscovich@ujf-grenoble.fr

1 Institut Joseph Fourier, Université Grenoble I, Saint-Martin-d'Hères, France

2 Departamento de Matemática, FCEyN, Universidad de Buenos Aires, Buenos Aires, Argentina

3 CONICET, Buenos Aires, Argentina 


\section{Introduction}

The notion of spectral sequence is due to Leray in his article [17], and it was presented in more algebraic terms by Koszul in [10] (see also [11]). Later on, Massey introduced the definition of exact couples (see [20,21]), as a more systematic mechanism to produce spectral sequences. One of the main examples considered by Massey is an exact couple coming from a filtered complex. In this manner, any filtered complex gives rise to an exact couple, which in turn produces a spectral sequence (see for instance [24], Part I, or [28], Ch. 5). We shall refer to this spectral sequence as the canonical one associated to the filtration. According to [23], Leray's work was closer to what we would now call a multiplicative spectral sequence, i.e. a spectral sequence provided with a product turning each page of it into a dg algebra. We recall that the underlying algebra of each successive page of a multiplicative spectral sequence is obtained by taking cohomology on the dg algebra given by the previous page. Notice that each of these dg algebras is in fact given by taking the total construction of a bigraded complex with a differential of a specific bidegree (depending on the index of the page of the spectral sequence). We shall say in this case that any such dg algebra is provided with a compatible bigrading (of a certain type). In case the filtered complex is a dg algebra (or even an $A_{\infty}$-algebra) and the filtration is multiplicative, the standard procedure recalled previously produces a multiplicative spectral sequence. Furthermore, Massey studied in [22] the algebraic structure required on an exact couple in order to obtain the corresponding algebraic structure on the associated spectral sequence.

On the other hand, Lapin has constructed in [13] a multiplicative spectral sequence from a formal bigraded deformation of a dg algebra (or of an $A_{\infty}$-algebra) provided with a compatible bigrading. Following [15], this in particular can be applied to the formal bigraded deformation coming from a filtered dg algebra (or $A_{\infty}$-algebra). The aim of this article is to show that the multiplicative spectral sequence constructed by Lapin is isomorphic to the canonical one. Moreover, another objective of the article is to explain the interesting constructions by Lapin in more structural terms. We would like to remark that we also became interested in the problem for its applications to persistent homology (see [5]).

The article is organized as follows. In Sect. 2 we recall the basic ingredients of the two algebraic structures we will be dealing with: spectral sequences and $A_{\infty}$-algebras. We present a result involving exact couples and spectral sequences that in our opinion should be well-known among the experts (see Proposition 2.1), but for which we could not find any precise reference. In Sect. 3 we recall the basic definitions and facts about the deformation theory of $A_{\infty}$-algebras. This theory has been studied under some specific situations by Fialowski and Penkava in [1], and by $\mathrm{Wu}$ in his Master thesis [29], which we recall. Moreover, we introduce the notion of formal bigraded deformation of a dg algebra (or an $A_{\infty}$-algebra) provided with a compatible bigrading (see Definition 3.1). Roughly speaking, a formal bigraded deformation is like a formal deformation where the indeterminate $\hbar$ has bidegree $(-1,1)$ and the corresponding completion (i.e. the inverse limit) is taken in the category of bigraded vector spaces. In any case, we believe that the experts should be aware of all these definitions, but since a precise reference is lacking, we provide them for clarity and completeness. 
We shall present grosso modo the main result of that section (where it appears under the name of Theorem 3.7), which is just a reformulation of a result by Lapin. We remark that we have not explicitly stated some gradings in it for the sake of simplicity, so the reader is advised to see the detailed version of this result in the corresponding section.

Theorem 1.1 (Lapin) Let $k$ be a field, A be an $A_{\infty}$-algebra provided with a compatible bigrading, and $A_{\hbar}$ be a formal bigraded deformation of $A$. Let us consider $H^{\bullet}(A)$ provided with an $A_{\infty}$-algebra structure and $f_{\bullet}: H^{\bullet}(A) \rightarrow A$ a quasi-isomorphism of $A_{\infty}$-algebras given by Kadeishvili's theorem. Then there exists a formal bigraded deformation $H^{\bullet}(A)_{\hbar}$ of $H^{\bullet}(A)$ and a quasi-isomorphism $\tilde{f}_{\bullet}: H^{\bullet}(A)_{\hbar} \rightarrow A_{\hbar}$ of formal bigraded deformations of $A_{\infty}$-algebras, i.e. such that we have the commutative diagram

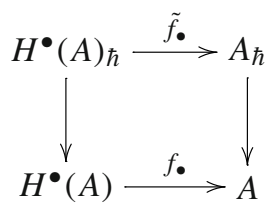

where the vertical maps are the canonical projections. Moreover, all these structures of formal bigraded deformations on $H^{\bullet}(A)$ are quasi-isomorphic.

Thus, roughly speaking, the previous result tells us that we may pull-back a formal bigraded deformation of an $A_{\infty}$-algebra to its cohomology such that both deformations are in fact quasi-isomorphic. We prove it by completely different methods to those used by Lapin in [13], Thm. 3.1 and Cor. 3.1, since ours use obstruction theory, instead of homological perturbation theory. The structure of the formal bigraded deformation of the cohomology is unique up to noncanonical quasi-isomorphism, a typical source of complications. Many of these constructions can be considered for algebras over cofibrant resolutions of Koszul operads, but we deal with the strongly homotopy associative case since it is the one considered by Lapin.

Section 4 includes how filtrations on $A_{\infty}$-algebras give rise to formal bigraded deformations in the previous sense, by adapting the well-known Rees algebra constructions (see Lemma 4.1). The rest of the section is devoted to establishing that any formal bigraded deformations in fact appears as the Rees $A_{\infty}$-algebra of a filtered $A_{\infty}$-algebra (see Proposition 4.2 ), although this is not really required to prove the main result of this work.

In the last section we recall the procedure introduced by Lapin in [13] to construct a multiplicative spectral sequence from a deformation of an $A_{\infty}$-algebra provided with a compatible bigrading. The previous construction is however rather hard to handle, so we provide a much more manageable way to deal with it, and we show that our procedure is "equivalent" to the one studied by Lapin. In any case, both constructions involve the notion of formal bigraded deformation of $A_{\infty}$-algebras, which is analysed in Sects. 3 and 4. In order to explain our results in more detail we describe very briefly the main ideas of the procedure given by Lapin and the one we give, but omitting the additional gradings for simplicity. Let $\left(A, m_{\bullet}\right)$ be an $A_{\infty}$-algebra provided with a compatible bigrading, and let $\left(A_{\hbar}, \tilde{m}_{\bullet}\right)$ be a formal bigraded deformation of $A$. On the 
one hand, the construction considered by Lapin consists of a collection $\left\{\left({ }^{r} A_{\hbar},{ }^{r} A\right)\right\}_{r \in \mathbb{N}}$, where ${ }^{r} A_{\hbar}$ is a formal bigraded deformation of ${ }^{r} A$, for each $r \in \mathbb{N}_{0},{ }^{0} A_{\hbar}=A_{\hbar}$ and ${ }^{0} A=A$. This sequence is defined recursively, and each term ${ }^{(r+1)} A_{\hbar}$ is deduced from ${ }^{r} A_{\hbar}$ in a two-step process: first, one takes cohomology on ${ }^{r} A$ and uses Theorem 1.1 to get a formal bigraded deformation of that cohomology; and finally, one changes the multiplications of the obtained deformation by a simple "shifting" process. It defines a multiplicative spectral sequence whose $r$-th page is ${ }^{r} A$, for $r \in \mathbb{N}$, that we will call the L-multiplicative spectral sequence (see Definition 5.1). On the other hand, we construct another collection $\left\{\left({ }^{r} A_{\hbar}^{\prime},{ }^{r} A^{\prime}\right)\right\}_{r \in \mathbb{N}}$, where ${ }^{r} A_{\hbar}^{\prime}$ is a formal bigraded deformation of ${ }^{r} A^{\prime}$, for each $r \in \mathbb{N}_{0},{ }^{0} A_{\hbar}^{\prime}=A_{\hbar}$ and ${ }^{0} A^{\prime}=A$. The terms of this sequence are completely explicit, without making use of Theorem 1.1. The first main result of this article, Theorem 5.8, roughly states that these two constructions are in fact equivalent, in the sense that, for each $r \in \mathbb{N}_{0}$, the corresponding formal bigraded deformations are quasi-isomorphic. For the sake of simplicity we will now present it but omitting some details, and we refer to the previously mentioned detailed statement.

Theorem 1.2 Let $k$ be a field, and $A_{\hbar}$ be a formal bigraded deformation of an $A_{\infty}$ algebra A provided with a compatible bigrading. Let us consider the collection of formal bigraded deformations of $A_{\infty}$-algebras $\left\{\left({ }^{r} A_{\hbar},{ }^{r} A\right)\right\}_{r \in \mathbb{N}}$ defined by Lapin and recalled in the previous paragraph, where ${ }^{0} A_{\hbar}=A_{\hbar}$ and ${ }^{0} A=A$. Let $\left({ }^{r} A_{\hbar}^{\prime},{ }^{r} A^{\prime}\right)$, for $r \in \mathbb{N}_{0}$, be the sequence of formal bigraded deformations of $A_{\infty}$-algebras we have introduced and also mentioned in the previous paragraph, where ${ }^{0} A_{\hbar}^{\prime}=A_{\hbar}$ and ${ }^{0} A^{\prime}=A$. Then, the formal bigraded deformations $\left({ }^{r} A_{\hbar},{ }^{r} A\right)$ and $\left({ }^{r} A_{\hbar}^{\prime},{ }^{r} A^{\prime}\right)$ are quasi-isomorphic for all $r \in \mathbb{N}$.

Our reason to introduce the new construction stated in the previous theorem is justified by the following fact, which we shall state in the particular case of dg algebras. If $A_{\hbar}$ is the formal bigraded deformation given by the Rees dg algebra $\operatorname{Re}_{F}{ }_{B}(B)$ of a filtered dg algebra $B$ (see Lemma 4.1), the dg algebra ${ }^{s} A_{\hbar}^{\prime}$ is canonically identified with the complex whose cohomology induces the upper terms of the $s$-th derived exact couple associated to the filtration of $B$, and ${ }^{s} A_{\hbar}^{\prime} /(\hbar)={ }^{s} A^{\prime}$ is canonically isomorphic to the corresponding lower term (see Proposition 2.1 and Fact 5.6). From this result and the previous theorem we derive that the $L$-multiplicative spectral sequence constructed by Lapin from a filtered dg algebra is isomorphic to the multiplicative spectral sequence canonically associated to the filtration (see Corollary 5.9), which is the main objective of the article. One interesting consequence of our detour is the corollary that any spectral sequence over a field comes from a filtration on a complex (see Remark 5.2).

We would like to thank Henning Krause, Mariano Suárez-Álvarez and Pablo Zadunaisky-Bustillos for several suggestions, and we would specially like to express our gratitude to Andrea Solotar for her encouragement and advice. Finally, we also thank the referee for all the comments and corrections.

\section{Preliminaries on basic algebraic structures}

We assume for the entire article that $k$ is a field. We shall also use the following convention throughout this article. If $V=\oplus_{n \in \mathbb{N}} V^{n}$ is a graded vector space over $k$, 
$V[1]$ is the graded vector space over $k$ whose $n$-th homogeneous component $V[1]^{n}$ is given by $V^{n+1}$, for all $n \in \mathbb{Z}$, and it is called the shift of $V$.

Given a collection of elements $\left\{v_{i}: i \in I\right\}$ in a vector space over $k$, a sum of the form $\sum_{(i \in I)} v_{i}$ will always mean that the previous collection is finitely supported, i.e. there exist a finite subset $I^{\prime} \subseteq I$ such that $a_{i}=0$, for all $i \in I \subseteq I^{\prime}$. This notation is used in order to distinguish the previous situation from the usual convergent infinite sums on a topological vector space or module. As usual, all unadorned tensor products $\otimes$ would be over $k$.

Finally, $\mathbb{N}$ will denote the set of (strictly) positive integers, whereas $\mathbb{N}_{0}$ will be the set of nonnegative integers. Similarly, for $N \in \mathbb{N}$ (resp., $N \in \mathbb{N}_{0}$ ), we denote by $\mathbb{N}_{\leq N}$ (resp., $\mathbb{N}_{0, \leq N}$ ) the set of positive (resp., nonnegative) integers less than or equal to $N$. Of course, similar notation could be used for other inequality signs.

\subsection{Basic setup on spectral sequences and exact couples}

We recall the basic setup for spectral sequences and exact couples coming from filtrations on a complex of vector spaces over $k$, with the main aim of fixing the notation we shall follow. A standard exposition on these subjects can be found in [24], Part I, or [28], Ch. 5, which we will partly follow. The main result we state, Proposition 2.1, is in our opinion well-known among the experts, but we could not find any reference for it.

We first introduce a the following convenient definition. Given a spectral sequence $\left(E_{r}\right)_{r \geq s}$ starting at $s$ and another spectral sequence $\left(E_{r}^{\prime}\right)_{r \geq s^{\prime}}$ starting at $s^{\prime} \geq s$, we shall say that they are compatible if the induced spectral sequence $\left(E_{r}\right)_{r \geq s^{\prime}}$ is isomorphic to $\left(E_{r}^{\prime}\right)_{r \geq s^{\prime}}$. In case the spectral sequences are multiplicative, the previous morphism is supposed to respect the corresponding product structures.

Let $\left(M, d_{M}\right)$ be a complex of vector spaces over $k$ with a decreasing filtration $\left\{F^{p} M\right\}_{p \in \mathbb{Z}}$ of the underlying graded vector space of $M$ such that $d_{M}\left(F^{p} M\right) \subseteq F^{p} M$, for all $p \in \mathbb{Z}$. For $s \in \mathbb{N}_{0}$, we define the complex of vector spaces over $k$

$$
{ }^{s} \mathcal{D}=\bigoplus_{p \in \mathbb{Z}}\left(\left(F^{p} M \cap d_{M}^{-1}\left(F^{p+s} M\right)\right) \otimes k . \hbar^{-p+s}\right) \subseteq M \otimes k\left[\hbar^{ \pm 1}\right],
$$

provided with the differential given by (the restriction of) $d_{M} \otimes \mathrm{id}_{k\left[\hbar^{ \pm 1}\right]} \hbar^{-s}$, and

$$
s_{\mathcal{E}}=\bigoplus_{p \in \mathbb{Z}}\left(\frac{F^{p} M \cap d_{M}^{-1}\left(F^{p+s} M\right)}{F^{p+1} M \cap d_{M}^{-1}\left(F^{p+1+s} M\right)}\right)
$$

with the induced differential by $d_{M}$. It sends the class $z+\left(F^{p+1} M \cap d_{M}^{-1}\left(F^{p+1+s} M\right)\right)$ to $d_{M}(z)+\left(F^{p+s+1} M \cap d_{M}^{-1}\left(F^{p+2 s+1} M\right)\right)$, for $z \in F^{p} M \cap d_{M}^{-1}\left(F^{p+s} M\right)$. Moreover, these complexes are bigraded by setting

$$
{ }^{s} \mathcal{D}^{p, q}=\left(F^{p+s} M^{p+q} \cap d_{M}^{-1}\left(F^{p+2 s} M^{p+q+1}\right)\right) \otimes k . \hbar^{-p}
$$


and

$$
{ }^{s} \mathcal{E}^{p, q}=\left(F^{p} M^{p+q} \cap d_{M}^{-1}\left(F^{p+s} M^{p+q+1}\right)\right) /\left(F^{p+1} M^{p+q} \cap d_{M}^{-1}\left(F^{p+1+s} M^{p+q+1}\right)\right) .
$$

The first bigrading convention is the one induced by that of $M \otimes k[\hbar \pm 1]$, where $M^{n}$ lies in bidegree $(0, n)$ and the bidegree of $\hbar$ is $(-1,1)$. Consider now the short exact sequence of complexes of vector spaces over $k$ of the form

$$
0 \rightarrow{ }^{s} \mathcal{D} \stackrel{s \tilde{i}}{\rightarrow}{ }^{s} \mathcal{D} \stackrel{s \tilde{j}}{\rightarrow}{ }^{s} \mathcal{E} \rightarrow 0
$$

where ${ }^{s} \tilde{i}$ is the morphism given by multiplication by $\hbar$, and ${ }^{s} \tilde{j}$ is the canonical projection multiplied by $\hbar^{-s}$. Note that ${ }^{s} \tilde{i}$ has bidegree $(-1,1)$ and ${ }^{s} \tilde{j}$ has bidegree $(s,-s)$ By considering the long exact sequence of cohomology groups of the previous short exact sequence, which may be rearranged as a triangle

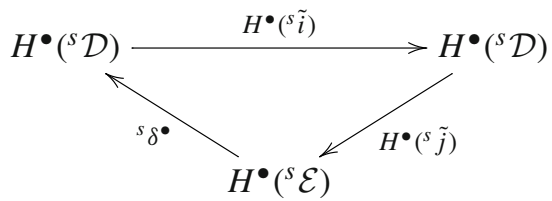

we get an exact couple of $s$-th type, where we recall that ${ }^{s} \delta^{\bullet}$ is the delta morphism obtained by means of the Snake lemma (see [28], Thm. 1.3.1, Example 5.9.3). It will be called the exact couple of $s$-th type associated to the filtration of the complex, and the corresponding spectral sequence starting at $(s+1)$ will be called the spectral sequence starting at $(s+1)$ associated to the filtration of the complex.

If $\left(M, d_{M}\right)$ is further a filtered dg algebra, adapting the ideas of [22], we introduce a product on (2.1) as follows. For $a \otimes \hbar^{-p+s}, b \otimes \hbar^{-q+s} \in{ }^{s} \mathcal{D}$, set $a \otimes \hbar^{-p+s} \cdot(b \otimes$ $\left.\hbar^{-q+s}\right)=a \cdot b \otimes \hbar^{-(p+q)+s}$. Provided with this product the complex ${ }^{s} \mathcal{D}$ becomes a dg algebra. The product on 2.2 is defined similarly. Moreover, it is straightforward to check that the exact couple (2.4) is multiplicative (see [22], Section 5, for the definition).

By the standard construction on spectral sequences coming from filtrations, one sees that for any $s, s^{\prime} \in \mathbb{N}_{0}$, the previously constructed spectral sequence starting at $(s+1)$ is compatible with the one starting at $\left(s^{\prime}+1\right)$. Moreover, the same arguments show the following stronger result, which should be well-known among the experts, but we give a sketch of the proof.

Proposition 2.1 Let $\left(M, d_{M}\right)$ be a filtered complex of vector spaces over $k$ as before. Then, the exact couple (2.4) of $s$-th type is isomorphic to the $s$-th derived exact couple of the one given by (2.4) for $s=0$. If $\left(M, d_{M}\right)$ is further a filtered dg algebra, the previous isomorphism is of multiplicative exact couples.

Proof Let us denote by $\left(E^{(s)}, D^{(s)}, \mathrm{i}^{(s)}, \mathrm{j}^{(s)}, \mathrm{k}^{(s)}\right)_{s \in \mathbb{N}_{0}}$ the collection of exact couples associated to the filtration of $M$, i.e. the one indexed by $s=0$ is the exact couple of 0 -th type associated to the filtration of $M$, and the one indexed by $s \in \mathbb{N}$ is the derived 
exact couple of the one indexed by $(s-1)$. Furthermore, by a very simple computation we have that

$$
\begin{aligned}
H^{\bullet}\left({ }^{s} \mathcal{D}\right) & =\bigoplus_{p \in \mathbb{Z}} \frac{F^{p} M \cap \operatorname{Ker}\left(d_{M}\right)}{d_{M}\left(F^{p-s} M \cap d_{M}^{-1}\left(F^{p} M\right)\right)} \otimes k \cdot \hbar^{-p+s} \\
& =\bigoplus_{p \in \mathbb{Z}} \frac{F^{p} M \cap \operatorname{Ker}\left(d_{M}\right)}{F^{p} M \cap d_{M}\left(F^{p-s} M\right)} \otimes k \cdot \hbar^{-p+s},
\end{aligned}
$$

where we have used the identity $d_{M}\left(F^{p-s} M \cap d_{M}^{-1}\left(F^{p} M\right)\right)=F^{p} M \cap d_{M}\left(F^{p-s} M\right)$. On the other hand, the image of the action of $\hbar^{s}$ on $H^{\bullet}\left({ }^{0} \mathcal{D}\right)$ (which coincides with the upper term $D^{(s)}$ of the $s$-th derived exact couple associated to the filtration of $B$ ) is

$$
\begin{aligned}
\hbar^{s} . H^{\bullet}\left({ }^{0} \mathcal{D}\right) & =\bigoplus_{p \in \mathbb{Z}} \frac{\left(F^{p+s} M \cap \operatorname{Ker}\left(d_{M}\right)\right)+d_{M}\left(F^{p} M\right)}{d_{M}\left(F^{p} M\right)} \otimes k \cdot \hbar^{-p} \\
& \simeq \bigoplus_{p \in \mathbb{Z}} \frac{F^{p+s} M \cap \operatorname{Ker}\left(d_{M}\right)}{F^{p+s} \cap d_{M}\left(F^{p} M\right)} \otimes k \cdot \hbar^{-p} \\
& =\bigoplus_{p \in \mathbb{Z}} \frac{F^{p} M \cap \operatorname{Ker}\left(d_{M}\right)}{F^{p} \cap d_{B}\left(F^{p-s} M\right)} \otimes k \cdot \hbar^{-p+s},
\end{aligned}
$$

where we have used the Second Isomorphism theorem in the second line. We have thus an isomorphism $t: D^{(s)} \rightarrow H^{\bullet}\left({ }^{s} \mathcal{D}\right)$ of bigraded vector spaces of bidegree $(0,0)$. In a similar manner (see the description of the $s$-th page $E^{(s)}$ of a spectral sequence coming from a filtration in [28], Chapter 5, Section 4), we have an isomorphism $u: E^{(s)} \rightarrow$ $H^{\bullet}\left({ }^{s} \mathcal{E}\right)$ of bigraded vector space of bidegree $(0,0)$. The two maps $t$ and $u$ give us an isomorphism between the exact couples of $s$-th type $\left(E^{(s)}, D^{(s)}, \mathrm{i}^{(s)}, \mathrm{j}^{(s)}, \mathrm{k}^{(s)}\right)$ and

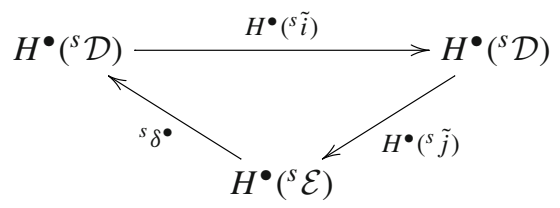

If $\left(M, d_{M}\right)$ is a filtered dg algebra, it is also straightforward to check that the isomorphism is of multiplicative exact couples. The proposition is thus proved.

Remark 2.2 Let $A$ be a filtered (graded complete) $A_{\infty}$-algebra (see Sect. 4 for the definition). It is trivial to see that the canonical spectral sequence associated to the underlying filtered complex of $A$ is in fact multiplicative.

\section{2 $A_{\infty}$-algebras and Kadeishvili's theorem}

The notion of $A_{\infty}$-algebra was introduced by Stasheff in [27] in his study of homotopy theory of loop spaces. We refer the reader to [25], Chapitre 3, or also [16], Chapitre 1, 
for standard references. Our particular sign and grading conventions are explained in detail in [4], Sects. 2 and 5, and even though they do not coincide with the references mentioned in the previous sentence, they agree with several others in the literature (see $[18,19]$ and references therein). We introduce however some extra bigrading conditions, which are not standard in the literature, but which are completely natural from the point of view of spectral sequences, and required in order to prove the main result of our article.

An $A_{\infty}$-algebra structure on a cohomological graded vector space $A$ is a collection of linear maps $m_{i}: A^{\otimes i} \rightarrow A$ for $i \in \mathbb{N}$ of cohomological degree $2-i$ satisfying the Stasheff identities $\mathrm{SI}(n)$ given by

$$
\sum_{(r, s, t) \in \mathcal{I}_{n}}(-1)^{r+s t} m_{r+1+t} \circ\left(\mathrm{id}_{A}^{\otimes r} \otimes m_{s} \otimes \mathrm{id}_{A}^{\otimes t}\right)=0
$$

for $n \in \mathbb{N}$, where $\mathcal{I}_{n}=\left\{(r, s, t) \in \mathbb{N}_{0} \times \mathbb{N} \times \mathbb{N}_{0}: r+s+t=n\right\}$. Given $N \in \mathbb{N}$, if $A$ is provided only with the morphisms $m_{i}$ for $i \in \mathbb{N}_{\leq N}$ and satisfy the Stasheff identities $\operatorname{SI}(n)$ for $n \in \mathbb{N}_{\leq N}$, we say that it is an $A_{N}$-algebra. Note that the first Stasheff identity SI(1) means that $m_{1}$ is a differential of $A$, so we may consider the cohomology $H^{\bullet}(A)$ given by the quotient $\operatorname{Ker}\left(m_{1}\right) / \operatorname{Im}\left(m_{1}\right)$.

Let $N \in \mathbb{N} \cup\{\infty\}$, and let $A$ be an $A_{N}$-algebra. If $N=\infty$, we define $\mathbb{N}_{\leq \infty}=$ $\mathbb{N}$. There exists a (not necessarily counitary) dg coalgebra $B_{N}(A)$, called the bar construction of $A$. If $N=\infty$, it is usually denoted just by $B(A)$. Its underlying graded coalgebra is given by the truncated tensor coalgebra $\oplus_{i \in \mathbb{N}_{\leq N}} A[1]^{\otimes i}$, where $A$ [1] denotes the shift of $A$. As usual, if $n \in \mathbb{N}_{\leq N}$ we will typically denote an element $s_{A}\left(a_{1}\right) \otimes \cdots \otimes s_{A}\left(a_{n}\right) \in A[1]^{\otimes n}$ in the form $\left[a_{1}|\ldots| a_{n}\right]$, where $a_{1}, \ldots, a_{n} \in A$, and $s_{A}: A \rightarrow A[1]$ is the canonical morphism of degree -1 whose underlying map of vector spaces over $k$ is the identity. The coproduct is given by the usual deconcatenation

$$
\Delta\left(\left[a_{1}|\ldots| a_{n}\right]\right)=\sum_{i=1}^{n-1}\left[a_{1}|\ldots| a_{i}\right] \otimes\left[a_{i+1}|\ldots| a_{n}\right] .
$$

Since $B_{N}(A)$ is a truncated tensor graded coalgebra, its differential $B_{N}$ can be defined as follows. It is the unique coderivation determined by $\pi_{1} \circ B_{N}$, where $\pi_{1}: B_{N}(A) \rightarrow$ $A[1]$ is the canonical projection (cf. [16], Lemme 1.1.2.2, and see [16], Section 1.2.2, pp. 29-30), such that this composition map is given by the sum $b=\sum_{i \in \mathbb{N}_{\leq N}} b_{i}$, where $b_{i}: A[1]^{\otimes i} \rightarrow A[1]$ is defined as $b_{i}=-s_{A} \circ m_{i} \circ\left(s_{A}^{\otimes i}\right)^{-1}$. In fact, Eq. (2.5) is precisely the condition for this coderivation to be a differential (cf. [16], Lemme 1.2.2.1, and see [16], Section 1.2.2, pp. 29-30).

An $A_{\infty}$-algebra is called (strictly) unitary if there is a map $\eta_{A}: k \rightarrow A$ of complete degree zero such that $m_{i} \circ\left(\mathrm{id}_{A}^{\otimes r} \otimes \eta_{A} \otimes \mathrm{id}_{A}^{\otimes t}\right)$ vanishes for all $i \neq 2$ and all $r, t \geq 0$ such that $r+1+t=i$. We shall usually denote the image of $1_{k}$ under $\eta_{A}$ by $1_{A}$, and call it the (strict) unit of $A$. Clearly, the flatness property may also be stated for strictly unitary $A_{\infty}$-algebras. We say that a unitary or nonunitary $A_{\infty}$-algebra is called minimal if $m_{1}$ vanishes. Note that all the previous definitions can be applied as well to $A_{N}$-algebras, for $N \in \mathbb{N}$. We see that a (unitary) dg algebra $\left(A, d_{A}, \mu_{A}\right)$ is a particular 
case of (unitary) $A_{\infty}$-algebra, where $m_{1}=d_{A}$ is the differential and $m_{2}=\mu_{A}$ is the product.

Given $s \in \mathbb{N}_{0}$, we say that an $A_{\infty}$-algebra $A$ has a compatible bigrading of $s$-th type if $A$ is provided with a bigrading $A=\oplus_{p, q \in \mathbb{Z}} A^{p, q}$ such that $A^{n}=\oplus_{p \in \mathbb{Z}} A^{p, n-p}$, and $m_{n}$ is a homogeneous morphism of bidegree $(2-n)(s,-s+1)$, for all $n \in \mathbb{N}$. If $A$ is unitary we further assume that $1_{A} \in A^{0,0}$. Note that if $A$ has a compatible bigrading (of $s$-th type), then the cohomology $H^{\bullet}(A)$ is provided with a canonically induced bigrading.

A morphism of $A_{\infty}$-algebras $f_{\bullet}: A \rightarrow B$ between two $A_{\infty}$-algebras $A$ and $B$ is a collection of morphisms of the underlying graded vector spaces $f_{i}: A^{\otimes n} \rightarrow B$ of cohomological degree $1-i$ for $i \in \mathbb{N}$ satisfying the Stasheff identities on morphisms $\operatorname{MI}(n)$ given by

$$
\sum_{(r, s, t) \in \mathcal{I}_{n}}(-1)^{r+s t} f_{r+1+t} \circ\left(\mathrm{id}_{A}^{\otimes r} \otimes m_{s}^{A} \otimes \mathrm{id}_{A}^{\otimes t}\right)=\sum_{q \in \mathbb{N}} \sum_{i \in \mathbb{N} q, n}(-1)^{w} m_{q}^{B} \circ\left(f_{i_{1}} \otimes \cdots \otimes f_{i_{q}}\right),
$$

for $n \in \mathbb{N}$, where $w=\sum_{j=1}^{q}(q-j)\left(i_{j}-1\right)$ and $\mathbb{N}^{q, n}$ is the subset of $\mathbb{N}^{q}$ of elements $\bar{i}=\left(i_{1}, \ldots, i_{q}\right)$ such that $|\bar{i}|=i_{1}+\cdots+i_{q}=n$. Given $N \in \mathbb{N}$, if $A$ and $B$ are only $A_{N}$-algebras, a morphism of $A_{N}$-algebras $f_{\bullet}: A \rightarrow B$ is a collection of morphisms of the underlying graded vector spaces $f_{i}: A^{\otimes n} \rightarrow B$ of cohomological degree $1-i$ for $i \in \mathbb{N}_{\leq N}$ satisfying the previous identities $\operatorname{MI}(n)$ for $n \in \mathbb{N}_{\leq N}$. If $A$ and $B$ are unitary $A_{\infty}$-algebras, the morphism $f_{\bullet}$ is called (strictly) unitary if $f_{1}\left(1_{A}\right)=1_{B}$, and for all $i \geq 2$ we have that $f_{i}\left(a_{1}, \ldots, a_{i}\right)$ vanishes if there exists $j \in\{1, \ldots, i\}$ such that $a_{j}=1_{A}$. Notice that $f_{1}$ is a morphism of complexes for the underlying structures on $A$ and $B$. We say that a morphism of (resp., unitary) $A_{\infty}$-algebras $f_{\bullet}: A \rightarrow B$ is a quasi-isomorphism if $f_{1}$ is a quasi-isomorphism of the underlying complexes. We say that a morphism $f_{\bullet}$ is strict if $f_{i}$ vanishes for $i \geq 2$. Note that all these definitions also apply to morphisms of $A_{N}$-algebras.

Given two (resp., unitary) $A_{\infty}$-algebras $A$ and $B$ provided with compatibles bigradings of $s$-th type, for some $s \in \mathbb{N}_{0}$, and a morphism $f_{\bullet}: A \rightarrow B$ of (resp., unitary) $A_{\infty}$-algebras, we say that $f_{\bullet}$ is compatible with the bigradings if $f_{n}$ is a homogeneous morphism of bidegree $(1-n)(s,-s+1)$, for all $n \in \mathbb{N}$.

Let $N \in \mathbb{N} \cup\{\infty\}$, and let $A$ and $A^{\prime}$ be two $A_{N}$-algebras. Given $f_{\bullet}: A \rightarrow A^{\prime}$ a morphism of $A_{N}$-algebras, it induces a morphism of dg coalgebras $B_{N}\left(f_{\bullet}\right): B_{N}(A) \rightarrow$ $B_{N}\left(A^{\prime}\right)$ between the bar constructions as follows. Taking into account that $B_{N}\left(A^{\prime}\right)$ is a truncated tensor coalgebra, such a morphism of graded coalgebras $F_{N}$ is completely determined by the composition $\pi_{1}^{\prime} \circ B_{N}\left(f_{\bullet}\right): B_{N}(A) \rightarrow A^{\prime}[1]$, where $\pi_{1}^{\prime}: B_{N}\left(A^{\prime}\right) \rightarrow A^{\prime}[1]$ denotes the canonical projection. The latter composition is given by a sum $\sum_{i \in \mathbb{N}_{\leq N}} F_{N, i}$, where $F_{N, i}: A[1]^{\otimes i} \rightarrow A^{\prime}[1]$, which we define to be $F_{N, i}=s_{A^{\prime}} \circ f_{i} \circ\left(s_{A}^{\otimes i}\right)^{-1}$, for $i \in \mathbb{N}_{\leq N}$. In fact, (2.6) is precisely the condition for this morphism to commute with the differentials (see [16], Section 1.2.2).

The following result is well-known and due to Kadeishvili (see [7], Thm. 1, for the case of dg algebras, or [8], Thm., for the case of plain $A_{\infty}$-algebras). We also consider some extra conditions about the bigradings which are suited for our study of spectral sequences. 
Theorem 2.3 Let $\left(A, m_{\bullet}\right)$ be an (resp., a unitary) $A_{\infty}$-algebra and $f_{1}: H^{\bullet}(A) \rightarrow A$ be the composition of a section of the canonical projection $\operatorname{Ker}\left(m_{1}\right) \rightarrow H^{\bullet}(A)$ and the inclusion $\operatorname{Ker}\left(m_{1}\right) \subseteq A$ (resp., satisfying that $\left.f_{1} \circ \eta_{H^{\bullet}(A)}=\eta_{A}\right)$. Then there exists a structure of (resp., unitary) $A_{\infty}$-algebra on $H^{\bullet}(A)$ given by $\left\{\bar{m}_{n}\right\}_{n \in \mathbb{N}}$ and a quasiisomorphism of (resp., unitary) $A_{\infty}$-algebras $f_{\bullet}: H^{\bullet}(A) \rightarrow$ A whose first component is $f_{1}$, such that $\bar{m}_{1}=0$ and $\bar{m}_{2}$ is the multiplication induced by $m_{2}$. Moreover, all these structures of (resp., unitary) $A_{\infty}$-algebras on $H^{\bullet}(A)$ are quasi-isomorphic. Any of these quasi-isomorphic (resp., unitary) $A_{\infty}$-structures will be called a model. Furthermore, if $A$ has a compatible bigrading of s-th type, for some $s \in \mathbb{N}_{0}$, then we may choose among these models one that is compatible with the induced bigrading on the cohomology $H^{\bullet}(A)$, such that the quasi-isomorphism of (resp., unitary) $A_{\infty}$ algebras $f_{\bullet}: H^{\bullet}(A) \rightarrow A$ can be chosen to be compatible with the bigradings. Any of these models on $H^{\bullet}(A)$ will be called compatible with the bigrading of $A$.

\section{Deformations of $A_{\infty}$-algebras}

The algebraic version of deformation theory for associative algebras was initiated by Gerstenhaber in [2], and was further extended for dg algebras by the mentioned author and C. Wilkerson in [3]. The theory for $A_{\infty}$-algebras is generalized more or less straightforward, and was studied by Fialowski and Penkava in [1], but also in the unpublished article [29] by Wu. There are however minor differences, as the conventions we used differ from the ones of the mentioned references, where they do not consider any completion as the ones appearing in this article. More precisely, we shall first recall in the following three paragraphs the standard definitions given in [1] about the deformation theory of an $A_{\infty}$-algebra over a finite dimensional $k$-algebra $R$, but focusing on the cases $R=k[\hbar] /\left(\hbar^{N+1}\right)$. In the subsequent two paragraphs we will explain some basic constructions of completed bigraded $k[\hbar]$-modules, where $\hbar$ has bidegree $(-1,1)$, and we shall introduce then the notion of formal bigraded deformation in Definition 3.1 (see also Remark 3.2). The rest of the section is devoted to proving Theorem 3.7, which was first proved by Lapin in [13], Thm. 3.1 and Cor. 3.1, but using different methods.

Let $\left(A, m_{\bullet}\right)$ be an $A_{\infty}$-algebra over $k$ provided with a compatible bigrading of $s$-th type, for some $s \in \mathbb{N}_{0}$, and let us consider the finite dimensional bigraded algebras $R=k[\hbar] /\left(\hbar^{(N+1)}\right)$, for $N \in \mathbb{N}$, where $\hbar$ has bidegree $(-1,1)$, called basic test bialgebras. In this brief section, a bigraded $R$-module will denote a symmetric bigraded $R$-bimodule. Following [1], an $R$-deformation of $A$ consists of the structure of $A_{\infty^{-}}$ algebra over $R$ on the (cohomologically) graded $R$-module $A \otimes R$ given by morphisms of $R$-modules

$$
m_{n}^{R}:(A \otimes R)^{\otimes_{R} n} \rightarrow A \otimes R
$$

of bidegree $(2-n)(s,-s+1)$, for all $n \in \mathbb{N}$, satisfying that $\left(\operatorname{id}_{A} \otimes \epsilon_{R}\right) \circ m_{n}^{R}$ restricted to $A^{\otimes n}$ coincides with $m_{n}$, for all $n \in \mathbb{N}$. We remark that $A^{\otimes n}$ is canonically identified with a subvector space of $(A \otimes R)^{\otimes} R^{n}$ by means of the map $a_{1} \otimes \cdots \otimes a_{n} \mapsto$ $\left(a_{1} \otimes 1_{R}\right) \otimes_{R} \cdots \otimes_{R}\left(a_{n} \otimes 1_{R}\right)$. Taking into account that $(A \otimes R)^{\otimes_{R} n} \simeq A^{\otimes n} \otimes R$, 
using the previous identification we may rephrase the last condition for the definition of deformation as $m_{n}^{R} \otimes_{R} \operatorname{id}_{k}=m_{n}$, for all $n \in \mathbb{N}$. If $A$ has a unit $1_{A}$, we say that the deformation preserves the unit if $1_{A} \otimes 1_{R} \in A \otimes R$ is a strict unit for the structure maps $\left\{m_{n}^{R}\right\}_{n \in \mathbb{N}}$. Given two $R$-deformations $A^{\prime}$ and $A^{\prime \prime}$ of the $A_{\infty}$-algebra $A$ provided of a compatible bigrading of $s$-th type, a (resp., strict) morphism of $R$-deformations from $A^{\prime}$ to $A^{\prime \prime}$ is a (resp., strict) morphism $f_{\bullet}$ of $A_{\infty}$-algebras over $R$ from $A^{\prime}$ to $A^{\prime \prime}$ such that $f_{\bullet} \otimes_{R} \operatorname{id}_{k}$ is the identity of $A$. We suppose the usual condition that $f_{n}$ has bidegree $(1-n)(s,-s+1)$, for all $n \in \mathbb{N}$. If $A$ has a unit, and $A^{\prime}$ and $A^{\prime \prime}$ preserve the unit, we shall further impose that $f_{\bullet}$ is a morphism of unitary $A_{\infty}$-algebras over $R$. Moreover, the $R$-deformation $A^{\prime}$ and $A^{\prime \prime}$ are said to be equivalent if there exists a morphism $f_{\bullet}: A^{\prime} \rightarrow A^{\prime \prime}$ of $R$-deformations of $A$ such that the induced morphism $F$ between the bar constructions $B\left(A^{\prime}\right)$ of $A^{\prime}$ and $B\left(A^{\prime \prime}\right)$ of $A^{\prime \prime}$ (where we remark that $A^{\prime}$ and $A^{\prime \prime}$ are (resp., unitary) $A_{\infty}$-algebras over $R$ ) is an isomorphism of dg coalgebras over $R$.

If $A$ and $B$ are two (resp., unitary) $A_{\infty}$-algebras over $k$, and $A_{R}$ and $B_{R}$ are two $R$-deformations of $A$ and $B$, respectively, a morphism from $A_{R}$ to $B_{R}$ is the data of a morphism $f_{\bullet}$ of (resp., unitary) $A_{\infty}$-algebras from $A$ to $B$, together with a morphism of (resp., unitary) $A_{\infty}$-algebras over $R$ given by a collection of maps

$$
\tilde{f}_{n}: A_{R}^{\otimes_{R} n} \rightarrow B_{R}
$$

for $n \in \mathbb{N}$, such that $\tilde{f}_{n} \otimes_{R} \operatorname{id}_{k}$ coincides with $f_{n}$, for all $n \in \mathbb{N}$, where we are making use of the isomorphisms $(A \otimes R)^{\otimes_{R} n} \simeq A^{\otimes n} \otimes R$ explained in the previous paragraph. We shall say that a morphism $\left(f_{\bullet}, \tilde{f}_{\bullet}\right)$ of $R$-deformations is a quasi-isomorphism if both $f_{\bullet}$ and $\tilde{f}_{\bullet}$ are quasi-isomorphisms.

Note that, if $A^{\prime}$ is an $R$-deformation of the (resp., unitary) $A_{\infty}$-algebra $A$ and let $T$ be a quotient of $R$, where both $R$ and $T$ are basic test bialgebras. Then $A^{\prime} \otimes_{R} T$ together with the maps $m_{n}^{R} \otimes_{R} \mathrm{id}_{T}$ is a $T$-deformation of $A$, called the deformation reduction to $T$ of $A^{\prime}$. Given the family of all basic test bialgebras $\left\{R_{i}=k[\hbar] /\left(\hbar^{(i+1)}\right)\right\}_{i \in \mathbb{N}}$ and a collection of (resp., unitary) $A_{\infty}$-algebras $\left\{A_{i}\right\}_{i \in \mathbb{N}}$ such that $A_{i}$ is an $R_{i}$-deformation of $A$, we say that this family is compatible if the deformation reduction of $A_{i+1}$ to $R_{i}$ given by the (resp., unitary) $A_{\infty}$-algebra $A_{i+1} \otimes_{R_{i+1}} R_{i}$ over $R_{i}$ and the $R_{i}$ deformation $A_{i}$ of $A$ are strictly isomorphic. A morphism from a compatible collection of deformations of (resp., unitary) $A_{\infty}$-algebras $\left\{A_{i}\right\}_{i \in \mathbb{N}}$ of $A$ to another compatible collection of deformations of (resp., unitary) $A_{\infty}$-algebras $\left\{B_{i}\right\}_{i \in \mathbb{N}}$ of $B$ is the data of a morphism $f_{\bullet}: A \rightarrow B$ of (resp., unitary) $A_{\infty}$-algebras together with collection of morphisms $\left\{\left(f_{\bullet}\right)_{i}\right\}_{i \in \mathbb{N}}$, where $\left(f_{\bullet}\right)_{i}$ is a morphism of (resp., unitary) $A_{\infty}$-algebras over $R_{i}$ from $A_{i}$ to $B_{i}$, such that $\left(f_{\bullet},\left(f_{\bullet}\right)_{i}\right)$ is a morphism of $R$-deformations from $A_{i}$ to $B_{i}$, for all $i \in \mathbb{N}$, satisfying that $\left(f_{\bullet}\right)_{i+1} \otimes_{R_{i+1}} \operatorname{id}_{R_{i}}$ coincides with $\left(f_{\bullet}\right)_{i}$, for all $i \in \mathbb{N}$. In this case we say that a morphism $\left(f_{\bullet},\left\{\left(f_{\bullet}\right)_{i}\right\}_{i \in \mathbb{N}}\right)$ of compatible families of deformation is a quasi-isomorphism if each $\left(f_{\bullet},\left(f_{\bullet}\right)_{i}\right)$ is a quasi-isomorphism of $R_{i}$-deformations, for all $i \in \mathbb{N}$.

We would like to provide the notion of formal deformation of a (unitary) $A_{\infty^{-}}$ algebra. Since our interest comes from spectral sequences where there is a bigrading, we shall deal with the case that our (unitary) $A_{\infty}$-algebra has a compatible bigrading. Most of the results we present here are analogous to the standard ones on formal 
deformation theory, which can be found for instance in [9], Ch. XVI, Sections 1-4. Some of the proofs are completely parallel to ones given there, and in that cases we only give the corresponding exact reference. Our difference relies that we also want to deal with the corresponding bigrading. For this reason, we will briefly explain the corresponding analogous setting of our interest. We will thus consider the commutative bigraded $k$-algebra in one indeterminate $k[\hbar]$, which is supposed to be bigraded by setting $\hbar$ in bidegree $(-1,1)$ (and $k$ with trivial bidegree). As before, we have the obvious augmentation map sending $\hbar$ to zero which will be denoted by $\epsilon_{k[\hbar]}$. Given any bigraded module $M$ over $k[\hbar]$, the inverse limit in the category of bigraded $k[\hbar]-$ modules

$$
\lim _{\leftarrow N} \operatorname{bgr}^{\sin } \otimes_{k[\hbar]} k[\hbar] /\left(\hbar^{N}\right)
$$

will be called the bigraded completion of $M$, and will be denoted by $\hat{M}^{\text {bgr }}$. There is a canonical morphism

$$
\tau_{M}^{\mathrm{bgr}}: M \rightarrow \hat{M}^{\mathrm{bgr}}
$$

of bigraded $k[\hbar]$-modules, and we will say that $M$ is bigraded complete if this latter map is an isomorphism. Also note that, given two bigraded $k[\hbar]$-modules $M$ and $N$, any homogeneous morphism (of bigraded $k[\hbar]$-modules) $f$ from $M$ to $N$ of bidegree $(p, q)$ automatically induces a homogeneous morphism (of bigraded $k[\hbar]$-modules) $\hat{f}^{\text {bgr }}$ from $\hat{M}^{\text {bgr }}$ to $\hat{N}^{\text {bgr }}$ of the same bidegree, and it is usually called the bigraded completion (morphism) of $f$. Given two bigraded modules $M$ and $N$ over $k[\hbar]$, the bigraded completed tensor product is defined as the bigraded completion of the usual tensor product $M \otimes_{k[\hbar]} N$, and it is denoted by

$$
M \hat{\otimes}_{k[\hbar]}^{\mathrm{bgr}} N
$$

Moreover, if $V=\oplus_{p, q \in \mathbb{Z}} V^{p, q}$ is a bigraded vector space over $k$, we may consider the bigraded $k[\hbar]$-module given by the tensor product $V \otimes k[\hbar]$, and we regard $V$ inside it via $v \mapsto v \otimes 1_{k[\hbar]}$. The bigraded completion of the previous $k[\hbar]$-module would be denoted by $V[[\hbar]]^{\text {bgr }}$ (which does not coincide in general with the previous usual tensor product). Notice that $V[[\hbar]]^{\text {bgr }}$ coincides with the inverse limit in the category of bigraded $k[\hbar]$ given by

$$
\lim _{\leftarrow N}^{\text {bgr }} V \otimes k[\hbar] /\left(\hbar^{N}\right) .
$$

We see that $V[[\hbar]]^{\text {bgr }}$ is given in more in more explicit terms by the bigraded vector space

$$
\bigoplus_{p, q \in \mathbb{Z}}\left(\prod_{r \in \mathbb{N}_{0}}\left(V^{p+r, q-r} \otimes k . \hbar^{r}\right)\right)
$$


together with the obvious action of $k[\hbar]$. Indeed, the inverse limit (3.1) is by very definition the bigraded vector space with homogeneous $(p, q)$-th component

$\lim _{\leftarrow N}\left(V \otimes k[\hbar] /\left(\hbar^{N}\right)\right)^{p, q}=\lim _{\leftarrow N}\left(\prod_{j=0}^{N} V^{p+j, q-j} \otimes k . \hbar^{j}\right)=\prod_{r \in \mathbb{N}_{0}}\left(V^{p+r, q-r} \otimes k . \hbar^{r}\right)$.

If $f: M^{\prime} \rightarrow M$ and $f: N^{\prime} \rightarrow N$ are two homogeneous morphisms of bigraded $k[\hbar]-$ modules of bidegrees $(p, q)$ and $\left(p^{\prime}, q^{\prime}\right)$, respectively, the bigraded completion of the morphism of $k[\hbar]$-modules

$$
f \otimes_{k[\hbar]} g: M^{\prime} \otimes_{k[\hbar]} N^{\prime} \rightarrow M \otimes_{k[\hbar]} N
$$

will be denoted by $f \hat{\otimes}_{k[\hbar]}^{\text {bgr }} g$. Any bigraded $k[\hbar]$-module $M$ isomorphic to $V[[\hbar]]^{\text {bgr }}$ for some bigraded vector space $V$ will be called $\hbar$-topologically free. Furthermore, it is easy to show that any bigraded $k[\hbar]$-module $M$ is $\hbar$-topologically free if and only if the canonical projection morphism $M \rightarrow M / \hbar$. $M$ of bigraded vector spaces has a section, it is bigraded complete and $\hbar$-torsion-free, i.e. if $\hbar . m=0$ for some $m \in M$, then $m=0$ (the exact same proof as in [9], Prop. XVI.2.4, applies here as well). Given two bigraded vector spaces $V$ and $W$ over $k$, we have a canonical (homogeneous) isomorphism

$$
(V \otimes W)[[\hbar]]^{\text {bgr }} \simeq V[[\hbar]]^{\text {bgr }} \hat{\otimes}_{k[\hbar]}^{\text {bgr }} W[[\hbar]]^{\text {bgr }}
$$

of bigraded $k[\hbar]$-modules of trivial bidegree (cf. [9], Prop. XVI.3.2). Indeed, by the description of $\hbar$-topologically free modules given in (3.2), we get that

$$
(V \otimes W)[[\hbar]]^{\mathrm{bgr}} \simeq \bigoplus_{p, q \in \mathbb{Z}}\left(\prod_{t \in \mathbb{N}_{0}}\left(\bigoplus_{p^{\prime}, q^{\prime} \in \mathbb{Z}}\left(V^{p^{\prime}, q^{\prime}} \otimes W^{p+t-p^{\prime}, q-t-q^{\prime}} \otimes k . \hbar^{t}\right)\right)\right),
$$

whereas

$$
\begin{aligned}
& V[[\hbar]]^{\text {bgr }} \otimes_{k[\hbar]} W[[\hbar]]^{\text {bgr }} \\
& \left.\simeq \bigoplus_{p^{\prime}, p^{\prime \prime}, q^{\prime}, q^{\prime \prime} \in \mathbb{Z}}\left(\left(\prod_{r \in \mathbb{N}_{0}}\left(V^{p^{\prime}+r, q^{\prime}-r} \otimes k \cdot \hbar^{r}\right)\right) \otimes_{k[\hbar]}\left(\prod_{s \in \mathbb{N}_{0}} W^{p^{\prime \prime}+s, q^{\prime \prime}-s} \otimes k . \hbar^{s}\right)\right)\right)
\end{aligned}
$$

so

$$
\begin{aligned}
& \left(V[[\hbar]]^{\mathrm{bgr}} \otimes_{k[\hbar]} W[[\hbar]]^{\mathrm{bgr}}\right) \otimes_{k[\hbar]} k[\hbar] /\left(\hbar^{(N+1)}\right) \\
& \simeq \bigoplus_{p, q \in \mathbb{Z}}\left(\prod_{t \in \mathbb{N}_{0, \leq N}}\left(\bigoplus_{p^{\prime}, q^{\prime} \in \mathbb{Z}}\left(V^{p^{\prime}, q^{\prime}} \otimes W^{p+t-p^{\prime}, q-t-q^{\prime}} \otimes k . \hbar^{t}\right)\right)\right),
\end{aligned}
$$


and the claim follows by taking inverse limit. Note that, if $M$ is a bigraded complete $k[\hbar]$-module the canonical map $\mathcal{H o m}_{k[\hbar]}\left(V[[\hbar]]^{\text {bgr }}, M\right) \rightarrow \mathcal{H} \operatorname{com}_{k}(V, M)$ given by restriction is an isomorphism of $k[\hbar]$-modules, where we recall that $\mathcal{H}$ om denotes the corresponding internal space of morphisms in the category of bigraded vector spaces (cf. [9], Prop. XVI.2.3. The exact same proof works here as well). Finally, we remark that, given a bigraded vector space $M$, the canonical map $M \otimes k[\hbar] /\left(\hbar^{N}\right) \rightarrow$ $M[[\hbar]]^{\text {bgr }} \otimes_{k[\hbar]} k[\hbar] /\left(\hbar^{N}\right)$ of right $k[\hbar] /\left(\hbar^{N}\right)$-modules induced by the morphism $m \otimes 1_{k[\hbar] /\left(\hbar^{N}\right)} \mapsto\left(m \otimes 1_{k[\hbar]}\right) \otimes_{k[\hbar]} 1_{k[\hbar] /\left(\hbar^{N}\right)}$ is an isomorphism.

Definition 3.1 A formal bigraded deformation $A_{\hbar}$ of an (resp., a unitary) $A_{\infty}$-algebra $\left(A, m_{\bullet}\right)$ over $k$ provided with a compatible bigrading of $s$-th type, for some $s \in \mathbb{N}_{0}$, is given by the $\hbar$-topologically free bigraded $k[\hbar]$-module $A[[\hbar]]^{\text {bgr }}$, where we recall that $\hbar$ has bidegree $(-1,1)$, provided with a collection of morphisms

$$
m_{n}^{\hbar}: A_{\hbar}^{\hat{\otimes}_{k[\hbar]^{n}}^{\mathrm{bgr}}} \rightarrow A_{\hbar}
$$

for $n \in \mathbb{N}$, of bidegree $(2-n)(s,-s+1)$ for $n \in \mathbb{N}$, such that the collection

$$
\left\{\left(A_{\hbar} \otimes_{k[\hbar]} k[\hbar] /\left(\hbar^{(N+1)}\right), m_{\bullet}^{\hbar} \otimes_{k[\hbar]} \operatorname{id}_{k[\hbar] /\left(\hbar^{(N+1)}\right)}\right)\right\}_{N \in \mathbb{N}}
$$

forms a compatible family of bigraded deformations of the (resp., unitary) $A_{\infty}$-algebra $A$ with respect to the family of test bigraded algebras $\left\{k[\hbar] /\left(\hbar^{(N+1)}\right)\right\}_{N \in \mathbb{N}}$, considered with the canonical projections. This implies that the restriction of the composition of $m_{n}^{\hbar}$ with $\left(\operatorname{id}_{A} \otimes \epsilon_{k[\hbar]}\right)$ to $A^{\otimes n}$ coincides with $m_{n}$, for all $n \in \mathbb{N}$.

Let $A_{\hbar}$ be a formal bigraded deformation of an (resp., a unitary) $A_{\infty}$-algebra $\left(A, m_{\bullet}\right)$ over $k$ provided with a compatible bigrading of $s$-th type, for some $s \in \mathbb{N}_{0}$. We remark that there is a canonical injection

$$
A^{\otimes n} \rightarrow A_{\hbar}^{\hat{\otimes}_{k[\hbar]}^{\mathrm{bgr}} n}
$$

of bigraded subvector spaces by means of the map $a_{1} \otimes \cdots \otimes a_{n} \mapsto\left(a_{1} \otimes 1_{k[\hbar]}\right) \otimes_{k[\hbar]}$ $\cdots \otimes_{k[\hbar]}\left(a_{n} \otimes 1_{k[\hbar]}\right)$. Note that by definition each of the deformations (3.3) are in fact (resp., unitary) $A_{\infty}$-algebras provided with a compatible bigrading of $s$-th type (when regarded over $k$ ).

Remark 3.2 We could have defined a formal bigraded deformation $A_{\hbar}$ of an (resp., a unitary) $A_{\infty}$-algebra $A$ as an $\hbar$-topologically free bigraded $k[\hbar]$-module provided with a structure of (resp., unitary) $A_{\infty}$-algebra over $k[\hbar]$, such that the underlying (resp., unitary) $A_{\infty}$-algebra over $k$ has a compatible bigrading of $s$-th type, and the reduction $A_{\hbar} \otimes_{k[\hbar]} k$ is strictly isomorphic to $A$. We note that in this case the structure maps $\tilde{m}_{i}$ of $A_{\hbar}$ should be given by maps

$$
\tilde{m}_{i}: A_{\hbar}^{\otimes_{k[\hbar]} i} \rightarrow A_{\hbar}
$$


instead of having as domain the completed tensor products appearing in the definition of formal bigraded deformation. However, since $A_{\hbar}$ is complete and $\tilde{m}_{i}$ is $k[\hbar]$-linear, it induces a structure map $m_{i}^{\hbar}$ as in the definition of the previous paragraph. A similar remark can be stated for the definition of morphisms in the next paragraph. We believe however that the proper definition should always rely on an inverse system of the type we considered, which is the usual manner one follows to handle the general case (e.g. if $\hbar$ is ungraded).

Remark 3.3 Our bigrading convention appearing in the definition of (formal bigraded deformations of) $A_{\infty}$-algebras should not make the reader think that we are handling a similar situation to that considered by Sagave in [26]. In that case the author is using a (different) bigrading to introduce a new notion - that of derived $A_{\infty}$-algebras-which allows him to prove a similar theorem to that of Kadeishvili over any commutative ring $K$ with unit. In his case, he gives however a minimal structure of derived $A_{\infty}$-algebra on a $K$-projective resolution of the cohomology of a given derived $A_{\infty}$-algebra. This result could be applied in our situation for $K=k[\hbar]$, which is roughly parallel to our Theorem 3.7, even though not precisely the same, for we get an honest $A_{\infty}$-algebra structure (over $K$ ), instead of a derived one.

If $A$ and $B$ are two (resp., unitary) $A_{\infty}$-algebras over $k$ provided with compatible bigradings of $s$-th type, for some $s \in \mathbb{N}_{0}$, and $A_{\hbar}$ and $B_{\hbar}$ are two formal bigraded deformations of $A$ and $B$, respectively, a morphism from $A_{\hbar}$ to $B_{\hbar}$ is the data of a morphism $f_{\bullet}$ of (resp., unitary) $A_{\infty}$-algebras from $A$ to $B$ compatible with the bigradings, together with a collection of maps

$$
\tilde{f}_{n}:\left(A_{\hbar}^{\mathrm{bgr}}\right)^{\hat{\otimes}_{k[\hbar]}^{\mathrm{bgr}}} \rightarrow B_{\hbar}^{\mathrm{bgr}},
$$

for $n \in \mathbb{N}$, of bidegree $(1-n)(s,-s+1)$ for $n \in \mathbb{N}$, such that the collection

$$
\left\{\left(\tilde{f}_{i} \otimes_{k[\hbar]} k[\hbar] /\left(\hbar^{(N+1)}\right)\right)_{i \in \mathbb{N}}\right\}_{N \in \mathbb{N}}
$$

forms a morphism of compatible collections a bigraded deformations of (resp., unitary) $A_{\infty}$-algebras, with respect to the family of test bigraded algebras given by $\left\{k[\hbar] /\left(\hbar^{(N+1)}\right)\right\}_{N \in \mathbb{N}}$, considered with the canonical projections.

Remark 3.4 Let $A_{\hbar}$ and $B_{\hbar}$ be formal bigraded deformations of the (resp., unitary) $A_{\infty}$-algebra $A$ and $B$, resp., where the two latter are provided with a compatible bigrading of $s$-th type, for some $s \in \mathbb{N}_{0}$. Let $\left(f_{\bullet}, \tilde{f}_{\bullet}\right)$ be a morphism of formal bigraded deformations from the deformation $A_{\hbar}$ of $A$ to the deformation $B_{\hbar}$ of $B$. It is a quasiisomorphism if and only if the morphism $\tilde{f}_{1}$ is a quasi-isomorphism between the corresponding underlying complexes over $k[\hbar]$. This follows from the easy fact, when writing out the definition that the induced morphism between the cohomology groups is an isomorphism, that the term corresponding to the zeroth power of $\hbar$ gives exactly the definition for $f_{1}=f_{1}^{0}$ to be a quasi-isomorphism.

We now state two lemmas required to ascertain the main theorem of this section. For their proof, see the "Appendix". 
Lemma 3.5 Let $N \in \mathbb{N}$, and let $\left(A,\left\{m_{i}\right\}_{i \in \mathbb{N}_{\leq N}}\right)$ and $\left(A^{\prime},\left\{m_{i}^{\prime}\right\}_{i \in \mathbb{N}_{\leq N}}\right)$ be two $A_{N^{-}}$ algebras such that $m_{1}^{\prime}=0$. Let $N^{\prime} \in \mathbb{N}$, and let $A_{\hbar^{\left(N^{\prime}+1\right)}}$ be a bigraded $k[\hbar] /\left(\hbar^{\left(N^{\prime}+1\right)}\right)$ deformation of $A$, and $A_{\hbar^{N^{\prime}}}^{\prime}$ a bigraded $k[\hbar] /\left(\hbar^{N^{\prime}}\right)$-deformation of $A^{\prime}$. Suppose given a morphism $\left\{\tilde{f}_{i}\right\}_{i \in \mathbb{N}_{\leq N}}$ of $A_{N}$-algebras over $k[\hbar] /\left(\hbar^{N^{\prime}}\right)$ such that the diagram

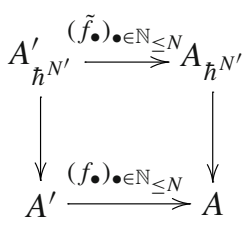

commutes. Suppose $f_{1}$ induces an injective map between the corresponding cohomology groups. Define the map from $\left(A^{\prime}\right)^{\otimes N}$ to A given by

$$
\begin{aligned}
U_{N}^{N^{\prime}}= & \sum_{(r, s, t) \in \mathcal{I}_{N}} \sum_{j \in \mathbb{N}_{0, \leq\left(N^{\prime}\right)}^{s}}(-1)^{r+s t} \tilde{f}_{r+1+t}^{j} \circ\left(\mathrm{id}_{A^{\prime}}^{\otimes r} \otimes\left(m^{\prime}\right)_{s}^{N^{\prime}-j} \otimes \mathrm{id}_{A^{\prime}}^{\otimes t}\right) \\
& -\sum_{q \in \mathbb{N}} \sum_{\bar{i} \in \mathbb{N} q, N} \sum_{j \in\left(\mathbb{N} q+1, N^{\prime}\right)^{*}}(-1)^{w} m_{q}^{j_{0}} \circ\left(\tilde{f}_{i_{1}}^{j_{1}} \otimes \cdots \otimes \tilde{f}_{i_{q}}^{j_{q}}\right),
\end{aligned}
$$

where $\bar{j}=\left(j_{0}, \ldots, j_{q}\right)$,

$$
\mathbb{N}_{0, \leq N^{\prime}}^{s}= \begin{cases}\mathbb{N}_{0, \leq N^{\prime}}, & \text { if } s \neq N \\ \mathbb{N}_{\leq N^{\prime}}, & \text { else, }\end{cases}
$$

and

$$
\left(\mathbb{N}^{q+1, N^{\prime}}\right)^{*}= \begin{cases}\mathbb{N}^{q+1, N^{\prime}}, & \text { if } q \neq 1, \\ \mathbb{N}^{2, N^{\prime}} \backslash\left\{\left(0, N^{\prime}\right)\right\}, & \text { else. }\end{cases}
$$

Then $m_{1}^{0} \circ U_{N}^{N^{\prime}}$ vanishes.

Lemma 3.6 Let $\left(M, d_{M}\right)$ be a complex of vector spaces over $k$, considered as an $A_{1}$ algebra, and provided with a compatible bigrading of $s$-th type, for some $s \in \mathbb{N}_{0}$. Let $M_{\hbar}$ be a formal bigraded deformation of $\left(M, d_{M}\right)$, which is an $A_{1}$-algebra over $k[\hbar]$ as well. Consider the cohomology $H^{\bullet}(M)$ of $\left(M, d_{M}\right)$, and take $f: H^{\bullet}(M) \rightarrow M$ the composition of a section of the canonical projection $\operatorname{Ker}\left(d_{M}\right) \rightarrow H^{\bullet}(M)$, which we suppose it exists, with the canonical inclusion $\operatorname{Ker}\left(d_{M}\right) \rightarrow M$. Suppose moreover we have $H^{\bullet}(M)_{\hbar}$ a formal bigraded deformation of $H^{\bullet}(M)$, which is just an $A_{1}$-algebra over $k[\hbar]$, together with a morphism $\tilde{f}: H^{\bullet}(M)_{\hbar} \rightarrow M_{\hbar}$ of complexes over $k[\hbar]$ such that 


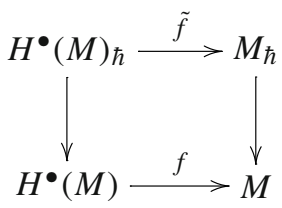

commutes. Then $\tilde{f}$ is a quasi-isomorphism (of complexes over $k[\hbar]$ ).

The following result is similar to the one proved by Lapin in [13], Thm. 3.1 and Cor. 3.1, but our proof follows the original Kadeishvili idea of obstruction theory, instead that of homological perturbation theory. As a detail, we do not need $k$ to be a field and we also take into account the bigrading we introduced previously.

Theorem 3.7 Let A be an (resp., a unitary) $A_{\infty}$-algebra over $k$ provided with a compatible bigrading of $s$-th type, for some $s \in \mathbb{N}_{0}$, and let $A_{\hbar}$ be a formal bigraded deformation of $A$. Let us consider $H^{\bullet}(A)$ provided with a model compatible with the bigrading of $A$ and $f_{\bullet}: H^{\bullet}(A) \rightarrow A$ a quasi-isomorphism of (resp., strictly unitary) $A_{\infty}$-algebras compatible with the corresponding bigradings. Then there exists a formal bigraded deformation $H^{\bullet}(A)_{\hbar}$ of $H^{\bullet}(A)$ and a quasi-isomorphism $\tilde{f}_{\bullet}: H^{\bullet}(A)_{\hbar} \rightarrow A_{\hbar}$ of formal bigraded deformations of (resp., strictly unitary) $A_{\infty}$-algebras, i.e. such that the underlying morphism of (resp., strictly unitary) $A_{\infty}$ algebras over $k$ is compatible with the bigradings, and we have the commutative diagram

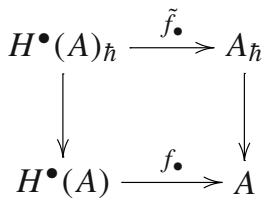

where the vertical maps are the canonical projections. Moreover, all these structures of formal bigraded deformations on $H^{\bullet}(A)$ are quasi-isomorphic.

Proof The proof will follow a similar pattern to the one of Kadeishvili's theorem we recalled at the end of the previous section, but with some subtleties coming from the deformation part.

Let us consider the following setting. We fix $N \in \mathbb{N}$, and we shall only regard the underlying $A_{N}$-algebra structures of $A$ and $H^{\bullet}(A)$. Moreover, given $N^{\prime} \in \mathbb{N}$, by taking a quotient modulo $\hbar^{\left(N^{\prime}+1\right)}$, we consider $A_{\hbar^{\left(N^{\prime}+1\right)}}=A_{\hbar} \otimes_{k[\hbar]} k[\hbar] /\left(\hbar^{\left(N^{\prime}+1\right)}\right)$ a $k[\hbar] /\left(\hbar^{\left(N^{\prime}+1\right)}\right)$-deformation of $A$. Assume we have defined a bigraded $k[\hbar] /\left(\hbar^{N^{\prime}}\right)$ deformation $H^{\bullet}(A)_{\hbar^{N^{\prime}}}$ of $H^{\bullet}(A)$ and a morphism $\left\{\tilde{f}_{i}\right\}_{i \in \mathbb{N}_{\leq N}}$ of $A_{N}$-algebras over $k[\hbar] /\left(\hbar^{N^{\prime}}\right)$ such that the diagram

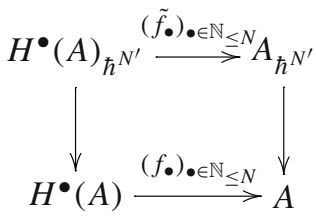


commutes. The bigrading assumption on the deformation $H^{\bullet}(A)_{\hbar^{N^{\prime}}}$ means that we may write $\left.\tilde{f}_{i}\right|_{H^{\bullet}(A) \otimes i}=\sum_{j=0}^{N^{\prime}-1} \tilde{f}_{i}^{j} \hbar^{j}$, where $\tilde{f}_{i}^{j}: H^{\bullet}(A)^{\otimes i} \rightarrow A$ is a homogeneous map of bidegree $(1-i)(s,-s+1)+(j,-j)$, for $i \in \mathbb{N}_{\leq N}$ and $j \in \mathbb{N}_{0, \leq\left(N^{\prime}-1\right)}$, where we recall that $\mathbb{N}_{0, \leq p}$ denotes the subset of $\mathbb{N}_{0}$ of nonnegative integers less than or equal to $p$. The previous commutativity means that $\tilde{f}_{i}^{0}=f_{i}$, for all $i \in \mathbb{N}_{\leq N}$. If $\left\{\tilde{m}_{i}\right\}_{i \in \mathbb{N}_{\leq N}}$ and $\left\{\tilde{\bar{m}}_{i}\right\}_{i \in \mathbb{N}_{\leq N}}$ denote the structure maps of the $A_{N}$-algebras over $k[\hbar] /\left(\hbar^{\left(N^{\prime}+1\right)}\right)$ and over $k[\hbar] /\left(\hbar^{N^{\prime}}\right)$ given by $A_{\hbar^{\left(N^{\prime}+1\right)}}$ and $H^{\bullet}(A)_{\hbar^{N^{\prime}}}$, resp., we will write $\left.\tilde{m}_{i}\right|_{A^{\otimes i}}=$ $\sum_{j=0}^{N^{\prime}} m_{i}^{j} \hbar^{j}$ and $\left.\tilde{\bar{m}}_{i}\right|_{H^{\bullet}(A) \otimes i}=\sum_{j=0}^{N^{\prime}-1} \bar{m}_{i}^{j} \hbar^{j}$, for $i \in \mathbb{N}_{\leq N}$, where $m_{i}^{j}: A^{\otimes i} \rightarrow A$ and $\bar{m}_{i}^{j}: H^{\bullet}(A)^{\otimes i} \rightarrow H^{\bullet}(A)$ are morphisms of bidegree $(2-i)(s,-s+1)+(j,-j)$, for $i \in \mathbb{N}_{\leq N}$, and $j \in \mathbb{N}_{0, \leq N^{\prime}}$ or $j \in \mathbb{N}_{0, \leq\left(N^{\prime}-1\right)}$, respectively. Suppose moreover that we have defined homogeneous maps $\bar{m}_{i}^{N^{\prime}}: H^{\bullet}(A)^{\otimes i} \rightarrow H^{\bullet}(A)$ of bidegree $(2-i)(s,-s+1)+\left(N^{\prime},-N^{\prime}\right)$, for $i \in \mathbb{N}_{\leq(N-1)}$, and morphisms $\tilde{f}_{i}^{N^{\prime}}: H^{\bullet}(A)^{\otimes i} \rightarrow A$ of bidegree $(1-i)(s,-s+1)+\left(N^{\prime},-N^{\prime}\right)$, for $i=1, \ldots, N-1$ such that they provide a morphism of $A_{N-1}$-algebras between the reductions $H^{\bullet}(A)_{\hbar^{\left(N^{\prime}+1\right)}}$ and $A_{\hbar^{\left(N^{\prime}+1\right)}}$. In order to prove the theorem it suffices to show the following statement: we may extend the previously defined morphism from a (also to be defined) $k[\hbar] /\left(\hbar^{\left(N^{\prime}+1\right)}\right)$ deformation $H^{\bullet}(A)_{\hbar^{\left(N^{\prime}+1\right)}}$ (extending $H^{\bullet}(A)_{\hbar^{N^{\prime}}}$ ) to $A_{\hbar^{\left(N^{\prime}+1\right)}}$, regarded as $A_{N}$-algebras over $k[\hbar] /\left(\hbar^{\left(N^{\prime}+1\right)}\right)$. Indeed, suppose that we proved the previous statement. We apply this procedure inductively as follows. First, for fixed $N=1$, we prove it for any $N^{\prime} \in \mathbb{N}$ by induction. Then, each time we increase the value of $N$ in one, we use only part of the already constructed structure maps $\left\{\tilde{\bar{m}}_{i}\right\}_{i \in \mathbb{N}_{\leq N}}$ and morphisms $\left\{\tilde{f}_{i}\right\}_{i \in \mathbb{N}_{\leq N}}$ : those involving terms $\bar{m}_{i}^{j}$ and $\tilde{f}_{i}^{j}$, with $j \leq\left(N^{\prime}-1\right)$, and apply the previous statement.

Let us now prove the statement. We consider the map from $H^{\bullet}(A)^{\otimes N}$ to $A$ of bidegree $(2-N)(s,-s+1)+\left(N^{\prime},-N^{\prime}\right)$ given by

$$
\begin{aligned}
U_{N}^{N^{\prime}}= & \sum_{(r, s, t) \in \mathcal{I}_{N}} \sum_{j \in \mathbb{N}_{0, \leq N^{\prime}}^{s}}(-1)^{r+s t} \tilde{f}_{r+1+t}^{j} \circ\left(\operatorname{id}_{H^{\bullet}(A)}^{\otimes r} \otimes \bar{m}_{s}^{N^{\prime}-j} \otimes \mathrm{id}_{H \cdot(A)}^{\otimes t}\right) \\
& -\sum_{q \in \mathbb{N}} \sum_{\bar{i} \in \mathbb{N} q, N} \sum_{\bar{j} \in\left(\mathbb{N} q+1, N^{\prime}\right)^{*}}(-1)^{w} m_{q}^{j_{0}} \circ\left(\tilde{f}_{i_{1}}^{j_{1}} \otimes \cdots \otimes \tilde{f}_{i_{q}}^{j_{q}}\right),
\end{aligned}
$$

where $\bar{j}=\left(j_{0}, \ldots, j_{q}\right)$,

$$
\mathbb{N}_{0, \leq N^{\prime}}^{s}= \begin{cases}\mathbb{N}_{0, \leq N^{\prime}}, & \text { if } s \neq N \\ \mathbb{N}_{\leq N^{\prime}}, & \text { else, }\end{cases}
$$

and

$$
\left(\mathbb{N}^{q+1, N^{\prime}}\right)^{*}= \begin{cases}\mathbb{N}^{q+1, N^{\prime}}, & \text { if } q \neq 1, \\ \mathbb{N}^{2, N^{\prime}} \backslash\left\{\left(0, N^{\prime}\right)\right\}, & \text { else. }\end{cases}
$$


By Lemma 3.5 we have that $m_{1}^{0} \circ U_{N}^{N^{\prime}}$ vanishes. We define $\bar{m}_{N}^{N^{\prime}}$ as minus the composition of $U_{N}^{N^{\prime}}$ and the canonical projection $\pi: \operatorname{Ker}\left(m_{1}\right) \rightarrow H^{\bullet}(A)$. By the very definition of $U_{N}^{N^{\prime}}$ we see that $U_{N}^{N^{\prime}}+f_{1}^{0} \circ \bar{m}_{N}^{N^{\prime}}=\left(\operatorname{id}_{\operatorname{Ker}\left(m_{1}\right)}-f_{1}^{0} \circ \pi\right) \circ U_{N}^{N^{\prime}}$ lies in the image of $m_{1}^{0}$, so there exists a morphism $f_{N}^{N^{\prime}}: H^{\bullet}(A)^{\otimes N} \rightarrow A$ (necessarily of bidegree $\left.(1-N)(s,-s+1)+\left(N^{\prime},-N^{\prime}\right)\right)$ such that

$$
U_{N}^{N^{\prime}}+f_{1}^{0} \circ \bar{m}_{N}^{N^{\prime}}=m_{1}^{0} \circ f_{N}^{N^{\prime}}
$$

which is just the term of the restriction to $H^{\bullet}(A)^{\otimes N}$ of the Stasheff identity on morphisms $\operatorname{MI}(N)$ of $A_{N}$-algebras over $k[\hbar] /\left(\hbar^{\left(N^{\prime}+1\right)}\right)$ which is multiplied by $\hbar^{N^{\prime}}$.

If we assumed $A$ is unitary, then a trivial verification shows that we may choose $\tilde{f}_{N}\left(\bar{a}_{1}, \ldots, \bar{a}_{N}\right)$ satisfying that it vanishes if there exists $j \in\{1, \ldots, N\}$ such that $\bar{a}_{j}=1_{H \bullet(A)}$. We have that $\tilde{\bar{m}}_{N}$ together with the previously considered multiplications $\tilde{\bar{m}}_{1}, \ldots, \tilde{\bar{m}}_{N-1}$ satisfy the Stasheff identity $\operatorname{SI}(N-1)$ as $A_{(N-1)}$-algebras over $k[\hbar] /\left(\hbar^{\left(N^{\prime}+1\right)}\right)$. Indeed, these Stasheff identities can be easily proved by considering the bar constructions of $H^{\bullet}(A)_{\hbar^{\left(N^{\prime}+1\right)}}$ and of $A_{\hbar^{\left(N^{\prime}+1\right)}}$ up to tensor degree $N$ with their corresponding coderivations $\tilde{\bar{B}}_{N}$ and $\tilde{B}_{N}$, constructed from $\tilde{\bar{m}}_{1}, \ldots, \tilde{\bar{m}}_{N}$ and from $\tilde{m}_{1}, \ldots, \tilde{m}_{N}$ respectively. The morphism identities up to tensor degree $N$ are tantamount to show that the morphism $\tilde{F}_{N}$ induced by $\tilde{f}_{1}, \ldots, \tilde{f}_{N}$ between the bar constructions of $H^{\bullet}(A)_{\hbar^{\left(N^{\prime}+1\right)}}$ and of $A_{\hbar^{\left(N^{\prime}+1\right)}}$ up to tensor degree $N$ satisfies that $\tilde{B}_{N} \circ \tilde{F}_{N}=\tilde{F}_{N} \circ \tilde{\bar{B}}_{N}$. The fact that $A_{\hbar^{\left(N^{\prime}+1\right)}}$ is an $A_{N}$-algebra over $k[\hbar] /\left(\hbar^{\left(N^{\prime}+1\right)}\right)$ tells us that $\tilde{B}_{N} \circ \tilde{B}_{N}=0$. Since $\tilde{f}_{1}$ is injective, for $\tilde{f}_{1}^{0}$ is also by definition (this follows from a straightforward computation), which in turn implies that $\tilde{F}_{N}$ is injective (see [6], Prop. 2.4.2), we get that $\tilde{\bar{B}}_{N} \circ \tilde{\bar{B}}_{N}=0$, which in turn implies the Stasheff identity $\operatorname{SI}(N)$ for $H^{\bullet}(A)_{\hbar^{\left(N^{\prime}+1\right)}}$.

If $A$ is assumed to be unitary, a trivial calculation shows that $\tilde{\bar{m}}_{N}\left(\bar{a}_{1}, \ldots, \bar{a}_{N}\right)$ vanishes if there exists $j \in\{1, \ldots, N\}$ such that $\bar{a}_{j}=1_{H \bullet(A)}$. Moreover, by Lemma 3.6, the map $\tilde{f}_{1}$ is a quasi-isomorphism. The last statement of the theorem also follows from the mentioned Lemma and the theorem is thus proved.

\section{Filtered deformations of $A_{\infty}$-algebras}

We shall present in this section the canonical construction of a formal bigraded deformation from a filtered $A_{\infty}$-algebra: the Rees $A_{\infty}$-algebra is a formal bigraded deformation of the associated graded $A_{\infty}$-algebra (see Lemma 4.1). We shall also prove that, under some graded completion assumption on the filtration, any formal bigraded deformation of an $A_{\infty}$-algebra arises in this way (see Proposition 4.2), even though this is not strictly necessary in order to prove the main result of this article. All these results are well-known in the case of filtered associative algebras, but a precise reference for the strongly homotopy associative ones is lacking to the best of our knowledge. We assume as usual that $k$ is a field, though this hypothesis is not strictly necessary for the main definitions of this section. 
A filtration on an $A_{\infty}$-algebra $\left(A, m_{\bullet}\right)$ is a decreasing filtration $\left\{F^{p} A\right\}_{p \in \mathbb{Z}}$ of the underlying graded vector space of $A$ satisfying the compatibility condition

$$
m_{n}\left(F^{p_{1}} A \otimes \cdots \otimes F^{p_{n}} A\right) \subseteq F^{p_{1}+\cdots+p_{n}} A
$$

for all $n \in \mathbb{N}$, and $p_{1}, \ldots, p_{n} \in \mathbb{Z}$. If $A$ has a unit we further assume that $1_{A} \in F^{0} A$. We shall assume that the filtration is exhaustive and graded complete (so Hausdorff), but not necessarily complete. We recall that the filtration is graded complete if $A$ is the inverse limit (in the category of graded vector spaces) of the inverse system given by the quotients $\left\{A / F^{p} A\right\}_{p \in \mathbb{Z}}$ provided with the obvious morphisms. Note that, given a filtered $A_{\infty}$-algebra $A$, the compatibility condition (4.1) tells us that the union $\cup_{p \in \mathbb{Z}} F^{p} A$ is also an $A_{\infty}$-algebra (such that the inclusion in $A$ is a strict morphism of $A_{\infty}$-algebras). Similarly, the structure maps $\left\{m_{n}\right\}_{n \in \mathbb{N}}$ of $A$ naturally induce an $A_{\infty}$ algebra $\left\{\hat{m}_{n}^{\text {gr }}\right\}_{n \in \mathbb{N}}$ on the graded completion construction of the underlying filtered graded vector space of $A$. Indeed, for any $p_{1}, \ldots, p_{n}$ consider the maps

$$
\left(\hat{A}^{\mathrm{gr}}\right)^{\otimes n} \rightarrow\left(\bigotimes_{j=1}^{n} A / F^{p_{j}} A\right) \rightarrow A / F^{p_{1}+\cdots+p_{n}} A
$$

given by the composition of the tensor product of canonical projections and the morphism induced by $m_{n}$. It is easy to show any pair of these maps for $p_{1}, \ldots, p_{n}$ and $p_{1}^{\prime}, \ldots, p_{n}^{\prime}$ satisfying that $p_{1}+\cdots+p_{n}=p_{1}^{\prime}+\cdots+p_{n}^{\prime}=p$ coincide, so we have the collection of morphisms of graded vector spaces

$$
q_{p}:\left(\hat{A}^{\mathrm{gr}}\right)^{\otimes n} \rightarrow A / F^{p} A
$$

indexed by $p$. This collection forms a system, i.e. given any pair of integers $p<p^{\prime}$, the composition of $q_{p^{\prime}}$ with the canonical projection $A / F^{p^{\prime}} A \rightarrow A / F^{p} A$ coincides with $q_{p}$. It thus induces a morphism of graded vector spaces $\left(\hat{A}^{\mathrm{gr}}\right)^{\otimes n} \rightarrow \hat{A}^{\mathrm{gr}}$, which we denoted by $\hat{m}_{n}^{\mathrm{gr}}$. The Stasheff identities are immediate. If $A$ has a unit $1_{A}$, its image under the canonical map $A \rightarrow \hat{A}^{\text {gr }}$ induces a unit on $\hat{A}^{\text {gr }}$.

Given a filtered $A_{\infty}$-algebra $\left(A, m_{\bullet}\right)$, the bigraded vector space $\operatorname{Gr}_{F}{ }_{A}(A)$ has a structure of $A_{\infty}$-algebra with structure maps $m_{n}^{\mathrm{gr}}$, for $n \in \mathbb{N}$, induced by those of $A$. It will be referred as the associated graded $A_{\infty}$-algebra. If $A$ has a unit $1_{A}$, its class (as an element of $F^{0} A / F^{1} A$ ) induces a unit on the associated graded $A_{\infty}$-algebra. Note that, if $A$ is a filtered dg algebra, the dg algebra $\operatorname{Gr}_{F}{ }_{A}(A)$ coincides with (2.2) for $s=0$. This $A_{\infty}$-algebra structure is in fact compatible with the bigrading of 0 -th type. Moreover, to a filtered $A_{\infty}$-algebra we may associate the Rees $A_{\infty}$-algebra $\operatorname{Re}_{F}{ }_{A}(A)$, given by

$$
\bigoplus_{p \in \mathbb{Z}}\left(F^{p} A \otimes k \cdot \hbar^{-p}\right) \subseteq A \otimes k\left[\hbar^{ \pm}\right]
$$

with structure maps

$$
m_{n}^{\mathrm{re}}\left(a_{1} \otimes \hbar^{-p_{1}}, \ldots, a_{n} \otimes \hbar^{-p_{n}}\right)=m_{n}\left(a_{1}, \ldots, a_{n}\right) \otimes \hbar^{-\left(p_{1}+\cdots+p_{n}\right)}
$$


for $n \in \mathbb{N}$. Note that the "polynomial part" is in some sense superfluous (and could be dropped from the definition) but it helps keeping track of the index $p$ coming from the filtration. If $A$ has a unit $1_{A}$, then $\operatorname{Re}_{F} \bullet A(A)$ has the strict unit $1_{A} \otimes \hbar^{0}$. We remark that, if $A$ is a filtered dg algebra, $\operatorname{Re}_{F}{ }_{A}(A)$ coincides with (2.1) for $s=0$.

On the other hand, the decreasing property of the filtration of $A$ tells us that $\operatorname{Re}_{F} \bullet A(A)$ has further the structure of bigraded $k[\hbar]$-module such that the inclusion (4.2) turns it into a $k[\hbar]$-submodule of $A \otimes k\left[\hbar^{ \pm}\right]$, provided with the regular action. Furthermore, note that if $A$ is unitary then $\operatorname{Re}_{F} \bullet A(A)$ has a canonical inclusion of the bigraded algebra $k[\hbar]$ given by $c \hbar^{p} \mapsto 1_{A} \otimes c \hbar^{p}$, for $c \in k$. By definition of the structure maps (4.3), it is direct to check that $\operatorname{Re}_{F}{ }_{A}(A)$ is in fact an $A_{\infty}$-algebra over $k[\hbar]$. We have moreover the following fact.

Lemma 4.1 Let $A$ be a filtered $A_{\infty}$-algebra as before. The Rees $A_{\infty}$-algebra $\operatorname{Re}_{F^{\bullet}}{ }_{A}(A)$ is a formal bigraded deformation of $\mathrm{Gr}_{F^{\bullet}}(A)$.

Proof For each $p \in \mathbb{Z}$ choose a graded subvector space $X^{p}$ of $F^{p} A$ such that $F^{p} A=$ $X^{p} \oplus F^{p+1} A$, for $k$ is a field. Since the filtration of $A$ is Hausdorff and $A$ is graded complete, the canonical morphism of graded vector spaces

$$
F^{p} A \rightarrow \prod_{q \in \mathbb{Z}_{\geq p}}{ }^{\mathrm{gr}} X^{q}
$$

is an isomorphism, where we recall that the codomain denotes the product in the category of graded vector spaces. Let us denote by $\pi_{q}^{p}: F^{p} A \rightarrow X^{q}$, for $p \leq q$, the composition of (any of) the previous morphism(s) and the canonical projection. Also denote by $\operatorname{proj}_{p}: F^{p} A \rightarrow F^{p} A / F^{p+1} A$ the canonical projection which identifies the vector space given by the codomain of the latter map and $X^{p}$. We may thus consider the $k$-linear map

$$
\begin{aligned}
\operatorname{Re}_{F}{ }_{A}(A) & \rightarrow \operatorname{Gr}_{F}{ }_{A}(A)[[\hbar]]^{\text {bgr }} \\
\sum_{(p \in \mathbb{Z})} a_{p} \otimes \hbar^{-p} & \mapsto \sum_{(p \in \mathbb{Z})} \sum_{q \in \mathbb{Z}_{\geq p}} \operatorname{proj}_{q}\left(\pi_{q}^{p}\left(a_{p}\right)\right) \otimes \hbar^{q-p},
\end{aligned}
$$

where we recall that the parentheses for the index $p$ means that the corresponding sums are finitely supported. The previous map is clearly well-defined [see (3.2)] and homogeneous $k[\hbar]$-linear of bidegree $(0,0)$. By the graded completion assumption on the filtration of $A$ and (3.2) we see that in fact it is bijective, so an isomorphism of bigraded $k[\hbar]$-modules.

Let $\left(B_{\hbar}, \tilde{m}_{\bullet}\right)$ be a formal bigraded deformation of an (resp., a unitary) $A_{\infty}$-algebra $\left(B, m_{\bullet}\right)$ provided with a compatible bigrading of 0 -th type. Given $p \in \mathbb{Z}$, set

$$
F^{p} B=\bigoplus_{p^{\prime} \geq p} \bigoplus_{q \in \mathbb{Z}} B^{p^{\prime}, q} .
$$

It defines a decreasing filtration on $B$, which is exhaustive and Hausdorff. Furthermore, if we regard $B$ as a graded vector space over $k$ for the total degree, the previous 
filtration is of graded vector spaces. We may thus consider the graded completion $\hat{B}^{\text {gr }}$ of the graded vector space $B$ with the previous filtration. By definition we see that the underlying graded vector space of $\hat{B}^{\text {gr }}$ is isomorphic to the subvector space of

$$
\bigoplus_{n \in \mathbb{Z}}\left(\prod_{p \in \mathbb{Z}} B^{p, n-p}\right)
$$

formed by the elements

$$
\hat{b}=\sum_{(n \in \mathbb{Z})} \sum_{p \in \mathbb{Z}} b_{p, n-p}
$$

where $b_{p, n-p} \in B^{p, n-p}$, such that for each $n \in \mathbb{Z}$ there exists $P_{n} \in \mathbb{Z}$ satisfying that $b_{p, n-p}$ vanishes for $p<P_{n}$. Moreover, for each $n \in \mathbb{Z}$, we define the map

$$
\mathrm{P}_{n}: \bigoplus_{n \in \mathbb{Z}}\left(\prod_{p \in \mathbb{Z}} B^{p, n-p}\right) \rightarrow\{-\infty\} \cup \mathbb{Z} \cup\{+\infty\},
$$

given by

$$
\mathrm{P}_{n}(\hat{b})=\inf \left\{p \in\{-\infty\} \cup \mathbb{Z} \cup\{+\infty\}: b_{p, n-p} \neq 0\right\}
$$

Note that the condition for $\hat{b}$ of being an element of $\hat{B}^{\text {gr }}$ implies that $\inf _{n \in \mathbb{Z}}\left(\mathrm{P}_{n}(\hat{b})\right)>$ $-\infty$. We shall denote the previous infimum by $\mathrm{P}(\hat{b})$, which defines a map with the same domain and codomain as each $\mathrm{P}_{n}$. Let us denote the canonical filtration of $\hat{B}^{\text {gr }}$ by $\left\{F^{p} \hat{B}^{\text {gr }}\right\}_{p \in \mathbb{Z}}$. Recall that we have an identification (as bigraded $k[\hbar]$-modules) between $B_{\hbar}$ and

$$
\bigoplus_{p, q \in \mathbb{Z}}\left(\prod_{r \in \mathbb{N}_{0}} B^{p+r, q-r} \otimes k . \hbar^{r}\right)
$$

Let $\iota: \hat{B}^{\text {gr }} \rightarrow B_{\hbar}$ be the map defined as

$$
\hat{b}=\sum_{(n \in \mathbb{Z})} \sum_{p \in \mathbb{Z}} b_{p, n-p} \mapsto \sum_{(n \in \mathbb{Z})} \sum_{r \in \mathbb{N}_{0}} b_{\mathrm{P}_{n}(\hat{b})+r, n-\mathrm{P}_{n}(\hat{b})-r} \hbar^{r}
$$

Note that $\iota$ is in fact a morphism of graded vector spaces from $\hat{B}^{\text {gr }}$ to the graded vector space $B_{\hbar}$ for the total degree.

On the other hand, since $\hbar$ has total degree 0 , the quotient $B_{\hbar} /(\hbar-1)$, which we shall denote by $\bar{B}$, is a graded vector space over $k$. Note that the kernel of the canonical projection $B_{\hbar} \rightarrow \bar{B}$ is given by $B_{\hbar}$. $(\hbar-1)$, formed by the set of elements given by $b .(\hbar-1)$, for $b \in B_{\hbar}$, and that $\bar{B}$ is an (resp., a unitary) $A_{\infty}$-algebra over $k$ with 
structure morphisms induce by those of $B_{\hbar}$. Indeed, given $n \in \mathbb{N}$, the map $\tilde{m}_{n}$ induces a map $\bar{m}_{n}: \bar{B}^{\otimes n} \rightarrow \bar{B}$, if given $b_{1}, \ldots, b_{n} \in B_{\hbar}$ such that there exists $i \in \mathbb{N}_{\leq n}$ with $b_{i}=b_{i}^{\prime}(\hbar-1)$, then $\tilde{m}_{n}\left(b_{1}, \ldots, b_{n}\right) \in B_{\hbar} .(\hbar-1)$. This last statement follows directly from the fact that $\tilde{m}_{n}$ is $k[\hbar]$-linear.

The composition of the map $\iota: \hat{B}^{\text {gr }} \rightarrow B_{\hbar}$ together with the canonical projection $B_{\hbar} \rightarrow \bar{B}$ defines a homogeneous morphism $\iota^{\prime}$ of graded vector spaces of degree zero. By taking into account the specific description of elements on both spaces we see that this composition map is in fact an isomorphism of graded vector spaces. We see that $\bar{B}$ is provided with a decreasing filtration of graded vector spaces given by the image of $\left\{F^{p} \hat{B}^{\text {gr }}\right\}_{p \in \mathbb{Z}}$ under $\iota^{\prime}$, which we denote by $\left\{F^{p} \bar{B}\right\}_{p \in \mathbb{Z}}$. It is exhaustive and complete, for the same occurs to $\hat{B}^{\text {gr }}$. Furthermore, the filtration $\left\{F^{\bullet} \bar{B}\right\}_{\bullet} \in \mathbb{Z}$ is respected by the (resp., unitary) $A_{\infty}$-algebra structure, so $\bar{B}$ becomes a filtered (resp., filtered unitary) $A_{\infty}$-algebra. In order to prove this last claim, take nonvanishing homogeneous elements $\bar{b}^{1} \in F^{p_{1}} \bar{B}, \ldots, \bar{b}^{i} \in F^{p_{i}} \bar{B}$ of degree $n_{1}, \ldots, n_{i}$, resp. In view of the fact that $\iota^{\prime}$ is a bijection, we have the corresponding elements $\hat{b}^{1} \in F^{p_{1}} \hat{B}^{\mathrm{gr}}, \ldots, \hat{b}^{i} \in F^{p_{i}} \hat{B}^{\mathrm{gr}}$ given by taking the inverse image under $\iota^{\prime}$ of the collection considered in the previous statement. Note that $\mathrm{P}\left(\hat{b}^{j}\right)=\mathrm{P}_{n_{j}}\left(\hat{b}^{j}\right) \geq p_{i}$, for all $j=1, \ldots, i$. We recall that $\bar{m}_{i}\left(\bar{b}^{1}, \ldots, \bar{b}^{i}\right)$ is the image of $\tilde{m}_{i}\left(\iota\left(\hat{b}^{1}\right), \ldots, \iota\left(\hat{b}^{i}\right)\right)$ under the canonical projection $B_{\hbar} \rightarrow \bar{B}$. By regarding the bidegrees of the elements $\iota\left(\hat{b}^{1}\right), \ldots, \iota\left(\hat{b}^{i}\right)$ we see that $\tilde{m}_{i}\left(\iota\left(\hat{b}^{1}\right), \ldots, \iota\left(\hat{b}^{i}\right)\right)$ is an element of bidegree $\left(\sum_{j=1}^{i} \mathrm{P}\left(\hat{b}^{j}\right), \sum_{j=1}^{i}\left(n_{j}-\mathrm{P}\left(\hat{b}^{j}\right)\right)\right)$ in $B_{\hbar}$, so it belongs to the image under $\iota$ of $F^{\sum_{j=1}^{i} \mathrm{P}\left(\hat{b}^{j}\right)} \hat{B}^{\text {gr }} \subseteq F^{\sum_{j=1}^{i} p_{j}} \hat{B}^{\text {gr }}$. Hence, we get that $\bar{m}_{i}\left(\bar{b}^{1}, \ldots, \bar{b}^{i}\right)$ lies in $F^{\sum_{j=1}^{i} p_{j}} \bar{B}$, as was to be shown.

We have the following result.

Proposition 4.2 Let $\left(B_{\hbar}, \tilde{m}_{\bullet}\right)$ be a formal bigraded deformation of an (resp., a unitary) $A_{\infty}$-algebra $\left(B, m_{\bullet}\right)$ provided with a compatible bigrading of 0 -th type. Consider the filtered (resp., filtered unitary) $A_{\infty}$-algebra $\bar{B}$ for the filtration $\left\{F^{\bullet} \bar{B}\right\}_{\bullet \in \mathbb{Z}}$ defined in the previous paragraph. Then the (resp., unitary) $A_{\infty}$-algebras over $k[\hbar]$ provided with compatible bigradings of 0 -th type given by $B_{\hbar}$ and the Rees $A_{\infty}$-algebra $\operatorname{Re}_{F} \bullet \bar{B}(\bar{B})$ are strictly isomorphic. Moreover, the formal bigraded deformations over $B$ they define are strictly isomorphic, such that the induced map on B is in fact the identity.

Proof Taking into account that $B_{\hbar}$ is a formal bigraded deformation of $B$, we shall write as usual $\left.\tilde{m}_{i}\right|_{B}=\sum_{j \in \mathbb{N}_{0}} m_{i}^{j} \hbar^{j}$. We will utilize the identification as filtered graded vector spaces between $\hat{B}^{\text {gr }}$ and $\bar{B}$ given by $\iota^{\prime}$. In particular we shall write an element $\bar{b} \in \bar{B}$ in the form

$$
\bar{b}=\sum_{(n \in \mathbb{Z})} \sum_{p \in \mathbb{Z}} b_{p, n-p},
$$

where $b_{p, n-p} \in B^{p, n-p}$, such that for each $n \in \mathbb{Z}$ there exists $P_{n} \in \mathbb{Z}$ satisfying that $b_{p, n-p}$ vanishes for $p<P_{n}$, and may also apply the maps $\mathrm{P}_{n}$ and $\mathrm{P}$ to elements of $\bar{B}$. By this identification, we shall that identify $F^{\bullet} \bar{B}$ with the respective filtration of $\hat{B}^{\text {gr }}$. So we may identify the $(p, q)$-th homogeneous component of the bigraded 
$k[\hbar]$-module $\operatorname{Re}_{F} \bullet \bar{B}(\bar{B})$ with $F^{p}\left(\hat{B}^{\text {gr }}\right)^{p+q}$. Under this identification, the structure map $m_{i}^{\text {re }}$ of $\operatorname{Re}_{F \cdot \bar{B}}(\bar{B})$ are given by composing

$$
\left.m_{i}^{\mathrm{re}}\right|_{\hat{B}_{\mathrm{gr}}}=\tilde{m}_{i} \circ \iota^{\otimes i}
$$

together with canonical projection $B_{\hbar} \rightarrow \bar{B}$.

We define an explicit map $\chi: \operatorname{Re}_{F} \bullet \bar{B}(\bar{B}) \rightarrow B_{\hbar}$ as follows. Let $\bar{b} \in F^{p} \bar{B}$ of degree $n$, which we regard by the identifications explained in the previous paragraph as

$$
\sum_{p^{\prime} \in \mathbb{Z}_{\geq p}} b_{p^{\prime}, n-p^{\prime}}
$$

The mapping $\chi$ is defined as the $k$-linear extension of the map

$$
\bar{b} \otimes \hbar^{-p} \mapsto \sum_{r \in \mathbb{N}_{0}} b_{p+r, n-p-r} \hbar^{r}
$$

Note that it is a morphism of bigraded $k[\hbar]$-modules, and that the restriction of $\chi$ to the homogeneous component of bidegree $(p, n-p)$ of $\operatorname{Re}_{F \bullet \bar{B}}(\bar{B})$ coincides with the $k[\hbar]$ linear map induced by the restriction to $F^{p}\left(\hat{B}^{\text {gr }}\right)^{n}$ of $\iota$. It also coincides with the map (4.4), after the obvious identification of (resp., unitary) $A_{\infty}$-algebras over $k$ provided with compatible bigradings between $B$ and $\operatorname{Gr}_{F} \hat{B}^{\text {gr }}\left(\hat{B}^{\text {gr }}\right)$. The map is clearly seen to be an isomorphism of graded $k[\hbar]$-modules, for its inverse is the $k$-linear extension of

$$
\sum_{r \in \mathbb{N}_{0}} b_{p+r, q-r} \hbar^{r} \mapsto\left(\sum_{r \in \mathbb{N}_{0}} b_{p+r, q-r}\right) \otimes \hbar^{-p} .
$$

We claim that $\chi$ is a strict morphism of (resp., unitary) $A_{\infty}$-algebras over $k[\hbar]$, i.e. $\tilde{m}_{i} \circ \chi^{\otimes_{k[\hbar]} i}=\chi \circ m_{i}^{\text {re }}$, for all $i \in \mathbb{N}$. This follows immediately from identity (4.5). Since we regard the Rees $A_{\infty}$-algebra $\operatorname{Re}_{F} \bullet \bar{B}(\bar{B})$ as a formal bigrading deformation of $B$ by means of the map (4.4), the comments in the previous paragraph tells us that $\chi: \operatorname{Re}_{F} \bullet \bar{B}(\bar{B}) \rightarrow B_{\hbar}$ gives in fact a strict isomorphism of formal bigraded deformations inducing the identity on $B$. The proposition is thus proved.

\section{Formal deformations of $A_{\infty}$-algebras and spectral sequences}

We shall consider in the forthcoming two paragraphs the following two definitions, which were studied by Lapin in [13], but were already known and had appeared in the literature in some particular cases. They will be described in terms of two functors, which we call $\mathrm{P}$ and $\mathrm{T}$. We moreover adapt them in our context of bigraded objects. From these constructions and from Theorem 3.7 we will then recall the procedure introduced by Lapin which produces a multiplicative spectral sequence from a formal bigraded deformation of an $A_{\infty}$-algebra. Since this construction is however rather hard to handle, we shall provide later an equivalent but much more manageable manner 
to deal with it. In particular, the new description will allow us to prove that, if the deformation comes from a filtration of the $A_{\infty}$-algebra, then the multiplicative spectral sequence introduced by Lapin is isomorphic to the canonical multiplicative spectral sequence associated to the filtration.

Let $\left(A, m_{\bullet}\right)$ be a minimal (resp., minimal unitary) $A_{\infty}$-algebra provided with a compatible bigrading of $s$-th type, for some $s \in \mathbb{N}_{0}$, and let $\left(A_{\hbar}, \tilde{m}_{\bullet}\right)$ be a formal bigraded deformation of $A$. The minimality assumption means that $m_{1}^{0}$ vanishes. We first define the projected dg algebra $\mathrm{P}\left(A_{\hbar}\right)$ of $A_{\hbar}$ as the (resp., unitary) dg algebra over $k$ whose underlying bigraded vector space is $A$, together with the multiplication $m_{2}$ and the differential $\tilde{m}_{1}^{1}$. It is a trivial verification that $\mathrm{P}\left(A_{\hbar}\right)$ is a (resp., unitary) dg algebra provided with a compatible bigrading of $(s+1)$-th type. We may in fact regard $\mathrm{P}$ as a functor from the category of formal bigraded deformations to the category of (resp., unitary) dg algebras. Let $A_{\hbar}$ and $B_{\hbar}$ be formal bigraded deformations of the (resp., unitary) $A_{\infty}$-algebras $A$ and $B$ provided with compatible bigradings of $s$-th type, for some $s \in \mathbb{N}_{0}$, and let $\left(f_{\bullet}, \tilde{f}_{\bullet}\right)_{\bullet \in \mathbb{N}}$ be a morphism of formal bigraded deformations from $A_{\hbar}$ to $B_{\hbar}$. Its image under $\mathrm{P}$ is the morphism of (resp., unitary) dg algebras from $\mathrm{P}\left(A_{\hbar}\right)$ to $\mathrm{P}\left(B_{\hbar}\right)$ given by $f_{1}$. Note that this functor sends quasi-isomorphisms to isomorphisms.

On the other hand, define the translated deformation $\mathrm{T}\left(A_{\hbar}\right)$ of $A_{\hbar}$ as the (resp., unitary) $A_{\infty}$-algebra over $k[\hbar]$ whose underlying bigraded $k[\hbar]$-module coincides with that of $A_{\hbar}$ but with structure maps given by $\tilde{m}_{i}^{\mathrm{T}}=\tilde{m}_{i} \hbar^{i-2}$. Note that $\tilde{m}_{1}^{\mathrm{T}}$ is well-defined because $m_{1}^{0}=0$. Furthermore, by the homogeneity of the definition on the structure maps, the Stasheff identity $\operatorname{SI}(n)$ of $\mathrm{T}\left(A_{\hbar}\right)$ is equivalent to the Stasheff identity $\operatorname{SI}(n)$ of $A_{\hbar}$ multiplied by $\hbar^{(n-3)}$. If $A$ is assumed to be unitary the identity axioms are straightforward. By a simple calculation the underlying (resp., unitary) $A_{\infty}$-algebra over $k$ of $\mathrm{T}\left(A_{\hbar}\right)$ has a compatible bigrading of $(s+1)$-th type, and it is in fact a formal bigraded deformation of the projected (resp., unitary) dg algebra $\mathrm{P}\left(A_{\hbar}\right)$. We can regard $\mathrm{T}$ as a functor from the category whose objects are formal bigraded deformations of (resp., unitary) $A_{\infty}$-algebras provided with a morphisms of formal bigraded deformations to itself. Indeed, let $A_{\hbar}$ and $B_{\hbar}$ be formal bigraded deformations of the (resp., unitary) $A_{\infty}$-algebras $A$ and $B$ provided with compatible bigradings of $s$-th type, for some $s \in \mathbb{N}_{0}$, and let $\left(f_{\bullet}, \tilde{f}_{\bullet}\right)_{\bullet \in \mathbb{N}}$ be a morphism of formal bigraded deformations from $A_{\hbar}$ to $B_{\hbar}$. The image of this morphism under $\mathrm{T}$ is given by the pair $\left(f_{1}, \mathrm{~T}(\tilde{f})\right)$ where we define the morphism $\mathrm{T}(\tilde{f})$ from $\mathrm{T}(A)$ to $\mathrm{T}(B)$, whose $i$-th component $\mathrm{T}(\tilde{f})_{i}$ is defined as $\tilde{f}_{i} \hbar^{i-1}$, for $i \in \mathbb{N}$. The Morphism identity $\operatorname{MI}(n)$ of $\mathrm{T}(\tilde{f}) \bullet$ is equivalent to the Morphism identity $\operatorname{MI}(n)$ of $\tilde{f}_{\bullet}$ multiplied by $\hbar^{(n-2)}$. If $A$ is assumed to be unitary the identity axioms are straightforward. Notice that this functor preserves quasi-isomorphisms.

Note now that we may combine Theorem 3.7 with the preceding definitions to produce a collection deformation as follows. Suppose $\left({ }^{0} A,{ }^{0} m_{\bullet}\right)$ is an (resp., a unitary) $A_{\infty}$-algebra provided with a compatible bigrading of 0 -th type, and let $\left({ }^{0} A_{\hbar},{ }^{0} \tilde{m}_{\bullet}\right)$ be a formal bigraded deformation of ${ }^{0} A$. By the previous theorem, we have a quasiisomorphic deformation given by the (resp., unitary) $A_{\infty}$-algebra $\left(H^{\bullet}\left({ }^{0} A\right),{ }^{0} \bar{m}_{\bullet}\right)$, also provided with a compatible bigrading of 0 -th type, and the formal bigraded deformation deformation $\left(H^{\bullet}\left({ }^{0} A\right)_{\hbar},{ }^{0} \tilde{\bar{m}}_{\bullet}\right)$, which satisfies by construction that ${ }^{0} \bar{m}_{1}^{0}=0$, so 
we may consider the new formal bigraded deformation $\mathrm{T}\left(H^{\bullet}\left({ }^{0} A\right)_{\hbar}\right)$ of the projected (resp., unitary) dg algebra $\mathrm{P}\left(H^{\bullet}\left({ }^{0} A\right)_{\hbar}\right)$, which has a compatible bigrading of first type. Let us call them ${ }^{1} A_{\hbar}$ and ${ }^{1} A$, respectively. By iteration we obtain thus a collection of (resp., unitary) dg algebras $\left\{{ }^{r} A\right\}_{r \in \mathbb{N}}$, such that ${ }^{r} A$ is provided with a compatible bigrading of $r$-th type together with a formal bigraded deformation ${ }^{r} A_{\hbar}$, for each $r \in \mathbb{N}$, and they satisfy that $\mathrm{T}\left(H^{\bullet}\left({ }^{r} A\right)_{\hbar}\right)={ }^{(r+1)} A_{\hbar}$ and $\mathrm{P}\left(H^{\bullet}\left({ }^{r} A\right)_{\hbar}\right)={ }^{(r+1)} A$, where $H^{\bullet}\left({ }^{r} A\right)_{\hbar}$ is the formal bigraded deformation of $H^{\bullet}\left({ }^{r} A\right)$ given by Theorem 3.7. We note that the collection of (resp., unitary) dg algebras $\left\{{ }^{r} A\right\}_{r \in \mathbb{N}}$ is in fact a multiplicative spectral sequence.

Definition 5.1 (Lapin) Let $\left({ }^{0} A,{ }^{0} m_{\bullet}\right)$ be an (resp., a unitary) $A_{\infty}$-algebra provided with a compatible bigrading of 0 -th type, and let $\left({ }^{0} A_{\hbar},{ }^{0} \tilde{m}_{\bullet}\right)$ be a formal bigraded deformation of ${ }^{0} A$. A spectral sequence $\left\{{ }^{r} A\right\}_{r \in \mathbb{N}}$ constructed as in the previous paragraph will be called the $L$-multiplicative spectral sequence associated to the formal bigraded deformation ${ }^{0} A_{\hbar}$ of ${ }^{0} A$. The collection of formal bigraded deformations produced will be called the associated family of formal bigraded deformations.

It would be an interesting question to precisely translate some of the typical properties that the $L$-multiplicative spectral sequence may fulfil (e.g. vanishing patterns at a particular step) in terms of the properties of a model structure on the deformation ${ }^{1} A_{\hbar}$.

Remark 5.2 Suppose $\left({ }^{0} A,{ }^{0} d\right)$ is a complex of vector spaces, regarded as an $A_{\infty^{-}}$ algebra with vanishing multiplications $m_{i}$, for $i \in \mathbb{N}_{\geq 2}$, provided with a compatible bigrading of 0 -th type, and let $\left({ }^{0} A_{\hbar},{ }^{0} \tilde{m}_{\bullet}\right)$ be a formal bigraded deformation of ${ }^{0} A$. By dropping if necessary all the multiplications ${ }^{0} \tilde{m}_{i}$, for $i \in \mathbb{N}_{\geq 2}$, we shall view ${ }^{0} A_{\hbar}$ as a complex over $k[\hbar]$, which gives a formal bigraded deformation of the complex ${ }^{0} A$. By forgetting if necessary at each step of the procedure explained in the previous paragraph all the involved multiplications indexed by $i \in \mathbb{N}_{\geq 2}$, for we consider only deformations of the differentials, one obtains a spectral sequence $\left\{{ }^{r} A\right\}_{r \in \mathbb{N}}$, which will be called the spectral sequence associated to the underlying deformation of complexes of the formal bigraded deformation ${ }^{0} A_{\hbar}$ of ${ }^{0} A$. By using a direct transference argument, Lapin has showed in [12], Prop. 3.2, that in fact any spectral sequence can be obtained in this way (though he does not considers any bigrading, this can be incorporated verbatim in his proof). By combining this together with Proposition 4.2 we can conclude that any spectral sequence over a field $k$ can be obtained from a filtration on a complex. This result does not seem to have been observed so far.

We want to observe that the procedure giving the $L$-multiplicative spectral sequence associated to the formal bigraded deformation ${ }^{0} A_{\hbar}$ of ${ }^{0} A$ is rather complicated to deal with, for the operations of taking cohomology at each step are in general very complicated to manage. We shall provide an equivalent but in our opinion simpler manner to handle with it. Let us explain this in more detail. Our new procedure will also consists of a sequence of formal bigraded deformations which we shall construct by means of a functor we shall now introduce and which we denote by D. Each of these formal bigraded deformations will be in fact quasi-isomorphic to the corresponding formal bigraded deformation in the definition of $A_{\infty}$-enhancement, which justifies our use for the word equivalence (see Proposition 5.5 and Theorem 5.8). 
Suppose $\left(A, m_{\bullet}\right)$ is an $A_{\infty}$ algebra over $k$ with a compatible bigrading of $s$-th type, for some $s \in \mathbb{N}_{0}$, and let $\left(A_{\hbar}, \tilde{m}_{\bullet}\right)$ be a formal bigraded deformation. We define in this case the $A_{\infty}$-algebra $\mathrm{D}\left(A_{\hbar}\right)$ over $k[\hbar]$ whose underlying bigraded vector space is

$$
\left\{a \in A_{\hbar}: \text { there exists } b \in A_{\hbar} \text { such that } \tilde{m}_{1}(a)=\hbar . b\right\},
$$

together with the structure maps $\tilde{m}_{i}^{\mathrm{D}}=\left.\hbar^{i-2} \tilde{m}_{i}\right|_{\mathrm{D}\left(A_{\hbar}\right)}$, for $i \in \mathbb{N}$. Note that the structure maps are well-defined, by definition of $\mathrm{D}\left(A_{\hbar}\right)$, i.e. the image of $\tilde{m}_{i}^{\mathrm{D}}$ lies inside $\mathrm{D}\left(A_{\hbar}\right)$. The previous construction is in fact functorial as stated in the following result.

Lemma 5.3 The operator D defined before induces a functor from the category of formal bigraded deformations of $A_{\infty}$-algebras provided with a compatible bigrading of $s$-th type, for some $s \in \mathbb{N}_{0}$, to itself. Indeed, if $A_{\hbar}$ and $B_{\hbar}$ are formal bigraded deformations of the (resp., unitary) $A_{\infty}$-algebras $A$ and $B$ provided with compatible bigradings of $s$-th type, for some $s \in \mathbb{N}_{0}$, and let $\left(f_{\bullet}, \tilde{f}_{\bullet}\right)_{\bullet \in \mathbb{N}}$ be a morphism of formal bigraded deformations from $A_{\hbar}$ to $B_{\hbar}$. The image of this morphism under $\mathrm{D}$ is the pair $\left(f_{1}, \mathrm{D}(\tilde{f})\right)$ with the morphism $\mathrm{D}(\tilde{f})$ from $\mathrm{T}(A)$ to $\mathrm{T}(B)$ whose $i$-th component $\mathrm{D}(\tilde{f})_{i}$ is given by $\left.\hbar^{i-1} \tilde{f}_{i}\right|_{\mathrm{D}\left(A_{\hbar}\right)}$, for $i \in \mathbb{N}$.

Proof We have to prove that $\mathrm{D}\left(A_{\hbar}\right)$ is in fact a formal bigraded deformation. Following the arguments given in the second paragraph of this section for proving that $\mathrm{T}\left(A_{\hbar}\right)$ is an $A_{\infty}$-algebra over $k[\hbar]$, we see that $\mathrm{D}\left(A_{\hbar}\right)$ is also. Furthermore, $\mathrm{D}\left(A_{\hbar}\right)$ is $\hbar$-torsionfree and bigraded complete, as one may conclude by a simple convergence argument (because $A_{\hbar}$ is bigraded complete and $\hbar . A_{\hbar} \subseteq D\left(A_{\hbar}\right)$ by definition), so it is an $\hbar$ topologically free bigraded $k[\hbar]$-module, by the comments in the second paragraph before Remark 3.2. By the explanation in the latter remark we see that $\mathrm{D}\left(A_{\hbar}\right)$ is in fact a formal bigraded deformation. In fact, it is easily seen that is a formal bigraded deformation of an $A_{\infty}$-algebra provided with a compatible bigrading of $(s+1)$-th type.

The previously defined functor satisfies the following properties.

Lemma 5.4 Let $\left(A, m_{\bullet}\right)$ be an $A_{\infty}$-algebra over $k$ with a compatible bigrading of $s$-th type, for some $s \in \mathbb{N}_{0}$, and let $\left(A_{\hbar}, \tilde{m}_{\bullet}\right)$ be a formal bigraded deformation. If $A$ is minimal, so $m_{1}$ vanishes, then $\mathrm{D}\left(A_{\hbar}\right)=\mathrm{T}\left(A_{\hbar}\right)$. On the other hand the functor $\mathrm{D}$ preserves quasi-isomorphisms.

Proof The first statement is trivial so we shall only prove the second one, which is in fact a direct computation, but we provide it nonetheless for clarity. Suppose that $\left(f_{\bullet}, \tilde{f}_{\bullet}\right)_{\bullet \in \mathbb{N}}$ is a quasi-isomorphism of formal bigraded deformations from $\left(A_{\hbar}, \tilde{m}_{\bullet}\right)$ to $\left(A_{\hbar}^{\prime}, \tilde{m}_{\bullet}^{\prime}\right)$. To prove that $\mathrm{D}(\tilde{f})_{1}$ induces an injective map in cohomology means that given $a \in A_{\hbar}$ such that $\tilde{m}_{1}(a)=0$ (or, equivalently, $a \in \mathrm{D}\left(A_{\hbar}\right)$ such that $\tilde{m}_{1}^{\mathrm{D}}(a)=0$ ), and given $a^{\prime} \in D\left(A_{\hbar}^{\prime}\right)$ such that $\tilde{f}_{1}(a)=\hbar^{-1} \tilde{m}_{1}^{\prime}\left(a^{\prime}\right)$, there exists $\alpha \in \mathrm{D}\left(A_{\hbar}\right)$ such that $a=\hbar^{-1} \tilde{m}_{1}(\alpha)$. The equality $\tilde{f}_{1}(a)=\hbar^{-1} \tilde{m}_{1}^{\prime}\left(a^{\prime}\right)$ can be rewritten as

$$
\tilde{m}_{1}^{\prime}\left(a^{\prime}\right)=\hbar \tilde{f}_{1}(a)=\tilde{f}_{1}(\hbar a)
$$


Now, since $\tilde{f}_{1}$ is a quasi-isomorphism from $A_{\hbar}$ to $A_{\hbar}^{\prime}$, we see that there exists $\alpha \in A_{\hbar}$ such that $\hbar a=\tilde{m}_{1}(\alpha)$. This in particular implies that $\alpha \in \mathrm{D}\left(A_{\hbar}\right)$ and the required equality, which proves the previously claimed injectivity. Let us now show that $\mathrm{D}(\tilde{f})_{1}$ induces a surjective map in cohomology. This is equivalent to prove that given $a^{\prime} \in A_{\hbar}^{\prime}$ such that $\tilde{m}_{1}^{\prime}\left(a^{\prime}\right)$ vanishes (or, equivalently, $a^{\prime} \in \mathrm{D}\left(A_{\hbar}^{\prime}\right)$ such that $\left.\left(\tilde{m}_{1}^{\prime}\right)^{\mathrm{D}}\left(a^{\prime}\right)=0\right)$, there exists $a \in A_{\hbar}$ such that $\tilde{m}_{1}(a)=0$ (or, equivalently, an element of $a \in \mathrm{D}\left(A_{\hbar}\right)$ satisfying that $\left.\tilde{m}_{1}^{\mathrm{D}}(a)=0\right)$ and an element $\alpha^{\prime} \in \mathrm{D}\left(A_{\hbar}^{\prime}\right)$ such that $\tilde{f}_{1}(a)=a^{\prime}+$ $\hbar^{-1} \tilde{m}_{1}^{\prime}\left(\alpha^{\prime}\right)$. However, since $\tilde{f}_{1}$ is a quasi-isomorphism from $A_{\hbar}$ to $A_{\hbar}^{\prime}$, we get that, given $a^{\prime}$ as before, there exists $a \in A_{\hbar}$ such that $\tilde{m}_{1}(a)=0$ (so a fortiori an element of $\mathrm{D}\left(A_{\hbar}\right)$ ) and $\alpha^{\prime \prime} \in A_{\hbar}^{\prime}$ such that $\tilde{f}_{1}(a)=a^{\prime}+\tilde{m}_{1}^{\prime}\left(\alpha^{\prime \prime}\right)$. By taking $\alpha^{\prime}=\hbar \alpha^{\prime \prime}$, which clearly belongs to $\mathrm{D}\left(A_{\hbar}^{\prime}\right)$, we get the desired identity which shows the claimed surjectivity. The quasi-isomorphism property of $\mathrm{D}(f)_{1}^{0}$ follows directly (see Remark 3.4).

We obtain the following consequence of the previous results.

Proposition 5.5 Let $\left(A, m_{\bullet}\right)$ be an $A_{\infty}$-algebra over $k$ with a compatible bigrading of $s$-th type, for some $s \in \mathbb{N}_{0}$, and let $\left(A_{\hbar}, \tilde{m}_{\bullet}\right)$ be a formal bigraded deformation. The formal bigraded deformations $\mathrm{D}\left(A_{\hbar}\right)$ and $\mathrm{T}\left(H^{\bullet}(A)_{\hbar}\right)$ are in fact quasi-isomorphic.

Proof By Theorem 3.7, there exists a formal bigraded deformation $H^{\bullet}(A)_{\hbar}$, which is quasi-isomorphic to $A_{\hbar}$. Then the formal bigraded deformations $\mathrm{D}\left(H^{\bullet}(A)_{\hbar}\right)$ and $\mathrm{D}\left(A_{\hbar}\right)$ are also quasi-isomorphic. In view of the fact that $\mathrm{D}\left(H^{\bullet}(A)_{\hbar}\right)$ coincides with $\mathrm{T}\left(H^{\bullet}(A)_{\hbar}\right)$, for $H^{\bullet}(A)_{\hbar}$ is a deformation of a minimal $A_{\infty}$-algebra, the claim follows.

In particular, this implies that we may build the associated family of formal bigraded deformations (up to quasi-isomorphism) in a more simple fashion, by defining $\left({ }^{r} A_{\hbar}^{\prime},{ }^{r} \tilde{m}_{\bullet}^{\prime}\right)$, for $r \in \mathbb{N}_{0}$, as the application of the composition functor $\mathrm{D}^{r}$ to $A_{\hbar}$, where $\mathrm{D}^{0}$ is the identity functor. We shall call this collection of formal bigraded deformations the reduced associated family of formal bigraded deformations. We note furthermore that our construction is easier to handle, due to the fact that, for instance D produces $\mathrm{dg}$ algebra deformation out of dg algebra deformations, unlike the usual associated family of formal bigraded deformations.

Our main reason to introduce the functor D relies on the following simple property.

Fact 5.6 Let $B$ be a filtered (graded complete) $A_{\infty}$-algebra, and $A_{\hbar}=\operatorname{Re}_{F} \bullet_{B}(B)$ be the Rees $A_{\infty}$-algebra associated to $B$. Given $s \in \mathbb{N}_{0}$, the underlying complex of $D^{s}\left(A_{\hbar}\right) . \hbar^{s}$ coincides with the $d g k[\hbar]$-module provided with a compatible bigrading given in (2.1) for the filtered complex $B$, and the underlying complex of $D^{S}\left(A_{\hbar}\right) /(\hbar)$ coincides with the corresponding associated graded complex of $B$ given in (2.2). The previous identifications are also compatible with the corresponding product structures given by $m_{2}$.

Remark 5.7 The identifications in the previous fact allow us to introduce $A_{\infty}$-algebra structures on the the complexes (2.1) and (2.2).

From the previous proposition we also derive one of our main results. 
Theorem 5.8 Let $A_{\hbar}$ a formal bigraded deformation of an (resp. a unitary) $A_{\infty}$ algebra A provided with a compatible bigrading of 0-th type. Let us consider the collection of (resp., unitary) $A_{\infty}$-algebras $\left\{{ }^{r} A\right\}_{r \in \mathbb{N}_{0}}$, where ${ }^{r} A$ is provided with a compatible bigrading of $r$-th type, together with a formal bigraded deformation ${ }^{r} A_{\hbar}$, for each $r \in \mathbb{N}_{0}$, defined as follows. First, we set ${ }^{0} A_{\hbar}=A_{\hbar}$ and ${ }^{0} A=A$. Recursively, define ${ }^{(r+1)} A_{\hbar}=\mathrm{T}\left(H^{\bullet}\left({ }^{r} A\right)_{\hbar}\right)$ and ${ }^{(r+1)} A=\mathrm{P}\left(H^{\bullet}\left({ }^{r} A\right)_{\hbar}\right)$, where $H^{\bullet}\left({ }^{r} A\right)_{\hbar}$ is the formal bigraded deformation of $H^{\bullet}\left({ }^{r} A\right)$ given by Theorem 3.7. On the other hand, define $\left({ }^{r} A_{\hbar}^{\prime},{ }^{r} A^{\prime}\right)$, for $r \in \mathbb{N}_{0}$, where ${ }^{r} A_{\hbar}^{\prime}$ is the application of the composition functor $\mathrm{D}^{r}$ to $A_{\hbar}, \mathrm{D}^{0}$ denotes the identity functor, and ${ }^{r} A^{\prime}={ }^{r} A_{\hbar}^{\prime} /(\hbar)$. Then ${ }^{r} A^{\prime}$ is an $A_{\infty}$-algebra provided with a compatible bigrading of $r$-th type, and ${ }^{r} A_{\hbar}^{\prime}$ is a formal bigraded deformation of ${ }^{r} A^{\prime}$. Moreover, the formal bigraded deformations $\left({ }^{r} A_{\hbar},{ }^{r} A\right)$ and $\left({ }^{r} A_{\hbar}^{\prime},{ }^{r} A^{\prime}\right)$ are quasi-isomorphic for all $r \in \mathbb{N}$.

We obtain now the following result, which is the main objective of the article, as a direct consequence of Theorem 5.8, Proposition 2.1 and Fact 5.6.

Corollary 5.9 Let $B$ be a filtered (graded complete) $A_{\infty}$-algebra, and $A_{\hbar}=$ $\operatorname{Re}_{F} \bullet_{B}(B)$ be the Rees $A_{\infty}$-algebra associated to $B$, which is a deformation of the $A_{\infty}$ algebra $A=\operatorname{Gr}_{F}{ }_{B}(B)$ provided with a compatible bigrading of 0 -th type. Then the $L$-multiplicative spectral sequence associated to the deformation $A_{\hbar}$ of $A$ and recalled in Definition 5.1 is isomorphic to the canonical multiplicative spectral sequence associated to $B$.

\section{Appendix}

We shall provide in this last part the proofs of the lemmas mentioned in Sect. 3.

Proof of Lemma 3.5 Choose any homogeneous maps $\left(m^{\prime}\right)_{N}^{N^{\prime}}:\left(A^{\prime}\right)^{\otimes N} \rightarrow A^{\prime}$ of bidegree $(2-N)(s,-s+1)+\left(N^{\prime},-N^{\prime}\right)$ and $\tilde{f}_{N}^{N^{\prime}}:\left(A^{\prime}\right)^{\otimes N} \rightarrow A$ of bidegree $(1-N)(s,-s+1)+\left(N^{\prime},-N^{\prime}\right)$. By the explanations on the bar construction of $A_{N^{-}}$ algebras given in the third paragraph of Sect. 2.2, the maps $\left\{\tilde{m}_{i}^{\prime}\right\}_{i \in \mathbb{N}_{\leq N}}$ given by $\left.\tilde{m}_{i}^{\prime}\right|_{A^{\prime}}=$ $\sum_{j=0}^{N}\left(m^{\prime}\right)_{i}^{j} \hbar^{j}$ induce a coderivation $\tilde{B}_{N}^{\prime}$ on the tensor coalgebra $B_{N}\left(A_{\hbar^{\left(N^{\prime}+1\right)}}^{\prime}\right)$. As $A_{\hbar^{\left(N^{\prime}+1\right)}}^{\prime}$ is not an $A_{N}$-algebra over $k[\hbar] /\left(\hbar^{\left(N^{\prime}+1\right)}\right)$ this is in fact an abuse of notation, and we should probably write instead something like $\bar{T}_{k[\hbar] /\left(\hbar^{\left(N^{\prime}+1\right)}\right)}^{c, \leq N}\left(A_{\hbar^{\left(N^{\prime}+1\right)}}^{\prime}[1]\right)$, but we will allow our notation for the sake of simplicity, and because it is completely clear that we are not assuming that $\tilde{B}_{N}^{\prime} \circ \tilde{B}_{N}^{\prime}$ vanishes. However, since $A_{\hbar^{\left(N^{\prime}+1\right)}}^{\prime}$ is an $A_{N}$-algebra over $k[\hbar] /\left(\hbar^{N^{\prime}}\right),\left(\pi^{\prime}\right)_{1}^{j} \circ \tilde{B}_{N}^{\prime} \circ \tilde{B}_{N}^{\prime} \circ\left(\iota^{\prime}\right)_{p}^{0}$ vanishes for $p=1, \ldots, N-1$ and $j=0, \ldots, N^{\prime}-1$, where $\left(\iota^{\prime}\right)_{p}^{0}:\left(A^{\prime}[1]\right)^{\otimes p} \rightarrow B_{N}\left(A_{\hbar^{\prime}\left(N^{\prime}+1\right)}\right)$ denotes the canonical inclusion, and $\left(\pi^{\prime}\right)_{1}^{j}$, for $j=0, \ldots, N^{\prime}$, denotes the composition of the canonical projections $B_{N}\left(A_{\hbar^{\left(N^{\prime}+1\right)}}^{\prime}\right) \rightarrow A_{\hbar^{\left(N^{\prime}+1\right)}}^{\prime}$ and $A_{\hbar^{\left(N^{\prime}+1\right)}}^{\prime} \rightarrow A^{\prime}$, where the latter map is given by $\sum_{l=0}^{N^{\prime}} a_{l} \hbar^{l} \mapsto a_{j}$. We use the analogous notation without primes for $A$. By following the recipe on the bar construction of a morphism of $A_{N}$-algebras, the maps $\left\{\tilde{f}_{i}\right\}_{i \in \mathbb{N}_{\leq N}}$ induce a morphism of graded coalgebras $\tilde{F}_{N}$ from $B_{N}\left(A_{\hbar^{\left(N^{\prime}+1\right)}}^{\prime}\right)$ to 
$B_{N}\left(A_{\hbar^{\left(N^{\prime}+1\right)}}\right)$. Define $\tilde{U}_{N}^{N^{\prime}}$ as $s_{A} \circ U_{N}^{N^{\prime}} \circ\left(s_{A^{\prime}}^{\otimes N}\right)^{-1}$. It is direct to show that

$$
\begin{aligned}
\tilde{U}_{N}^{N^{\prime}}= & \sum_{(r, s, t) \in \mathcal{I}_{N}} \sum_{j \in \mathbb{N}_{0, \leq\left(N^{\prime}\right)}^{s}} \tilde{F}_{N, r+1+t}^{j} \circ\left(\mathrm{id}_{A^{\prime}}^{\otimes r} \otimes\left(b^{\prime}\right)_{s}^{N^{\prime}-j} \otimes \mathrm{id}_{A^{\prime}}^{\otimes t}\right) \\
& -\sum_{q \in \mathbb{N}} \sum_{\bar{i} \in \mathbb{N} q, N} \sum_{j \in\left(\mathbb{N} q+1, N^{\prime}\right)^{*}} b_{q}^{j_{0}} \circ\left(\tilde{F}_{N, i_{1}}^{j_{1}} \otimes \cdots \otimes \tilde{F}_{N, i_{q}}^{j_{q}}\right) .
\end{aligned}
$$

It is clear that that the vanishing of $m_{1}^{0} \circ U_{N}^{N^{\prime}}$ is tantamount to the vanishing of $b_{1}^{0} \circ \tilde{U}_{N}^{N^{\prime}}$. Moreover, it is easy to see that

$\tilde{U}_{N}^{N^{\prime}}=\pi_{1}^{N^{\prime}} \circ\left(\tilde{F}_{N+1} \circ \tilde{B}_{N+1}^{\prime}-\tilde{B}_{N+1} \circ \tilde{F}_{N+1}\right) \circ\left(\iota^{\prime}\right)_{N}^{0}-\tilde{F}_{N, 1}^{0} \circ\left(b^{\prime}\right)_{N}^{N^{\prime}}+b_{1}^{0} \circ \tilde{F}_{N, N}^{N^{\prime}} \cdot$

Then

$$
\begin{aligned}
b_{1}^{0} \circ \tilde{U}_{N}^{N^{\prime}} & =b_{1}^{0} \circ \pi_{1}^{N^{\prime}} \circ\left(\tilde{F}_{N} \circ \tilde{B}_{N}^{\prime}-\tilde{B}_{N} \circ \tilde{F}_{N}\right) \circ\left(\iota^{\prime}\right)_{N}^{0} \\
& =\tilde{B}_{N} \circ\left(\tilde{F}_{N} \circ \tilde{B}_{N}^{\prime}-\tilde{B}_{N} \circ \tilde{F}_{N}\right) \circ\left(\iota^{\prime}\right)_{N}^{0} \\
& =\tilde{B}_{N} \circ \tilde{F}_{N} \circ \tilde{B}_{N}^{\prime} \circ\left(\iota^{\prime}\right)_{N}^{0},
\end{aligned}
$$

where we have used in the first equality that $b_{1}^{0} \circ b_{1}^{0}=0$ and $b_{1}^{0} \circ \tilde{F}_{N, 1}^{0}=0$, for $m_{1}^{0} \circ m_{1}^{0}=0$ and $m_{1}^{0} \circ \tilde{f}_{1}^{0}=0$, and in the second equality that the image of $\left(\tilde{F}_{N} \circ\right.$ $\left.\tilde{B}_{N}^{\prime}-\tilde{B}_{N} \circ \tilde{F}_{N}\right) \circ\left(\iota^{\prime}\right)_{N}^{0}$ is included in the image of $\iota_{1}^{0} \hbar^{N^{\prime}}$, by the hypothesis that $\left\{\tilde{f}_{i}\right\}_{i \in \mathbb{N}_{\leq N}}$ is a morphism of $A_{N}$-algebras over $k[\hbar] /\left(\hbar^{N^{\prime}}\right)$. In the last equality we have used that $\tilde{B}_{N} \circ \tilde{B}_{N}=0$, for $A_{\hbar^{\left(N^{\prime}+1\right)}}$ is an $A_{N}$-algebra. Since the image of $\tilde{B}_{N}^{\prime} \circ\left(\iota^{\prime}\right)_{N}^{0}$ is included in $B_{N}\left(A^{\prime}\right)$, because $\left(b^{\prime}\right)_{1}^{0}$ vanishes, the restriction to $B_{N}\left(A_{\hbar^{N^{\prime}}}^{\prime}\right)$ of the morphism $\tilde{F}_{N}$ from $B_{N}\left(A_{\hbar^{\left(N^{\prime}+1\right)}}^{\prime}\right)$ to $B_{N}\left(A_{\hbar^{\left(N^{\prime}+1\right)}}\right)$ coincides with the morphism $\tilde{F}_{N}$ from $B_{N}\left(A_{\hbar^{N^{\prime}}}^{\prime}\right)$ to $B_{N}\left(A_{\hbar^{N^{\prime}}}\right)$, and $\tilde{F}_{N} \circ \tilde{B}_{N}^{\prime}=\tilde{B}_{N} \circ \tilde{F}_{N}$, we see that $b_{1}^{0} \circ \tilde{U}_{N}^{N^{\prime}}$ coincides with

$$
\tilde{B}_{N} \circ \tilde{F}_{N} \circ \tilde{B}_{N}^{\prime} \circ\left(\iota^{\prime}\right)_{N}^{0}=\tilde{F}_{N} \circ \tilde{B}_{N}^{\prime} \circ \tilde{B}_{N}^{\prime} \circ\left(\iota^{\prime}\right)_{N}^{0} .
$$

Note that $\tilde{B}_{N}^{\prime} \circ \tilde{B}_{N}^{\prime} \circ\left(\iota^{\prime}\right)_{N}^{0}=\pi_{1}^{N^{\prime}} \circ \tilde{B}_{N}^{\prime} \circ \tilde{B}_{N}^{\prime} \circ\left(\iota^{\prime}\right)_{N}^{0}$, because $A_{\hbar^{N^{\prime}}}^{\prime}$ is an $A_{N}$-algebra over $k[\hbar] /\left(\hbar^{N^{\prime}}\right)$ (see [16], Lemme B.1.1, a), and thus $b_{1}^{0} \circ \tilde{U}_{N}^{N^{\prime}}$ coincides with

$$
\tilde{F}_{N, 1}^{N^{\prime}} \circ\left(\pi_{1}^{N^{\prime}} \circ \tilde{B}_{N}^{\prime} \circ \tilde{B}_{N}^{\prime} \circ\left(\iota^{\prime}\right)_{N}^{0}\right)
$$

Choose any $\omega \in\left(A^{\prime}[1]\right)^{\otimes N}$, and consider $a^{\prime}=\left(\pi_{1}^{N^{\prime}} \circ \tilde{B}_{N}^{\prime} \circ \tilde{B}_{N}^{\prime} \circ\left(\iota^{\prime}\right)_{N}^{0}\right)(\omega)$. It is a cocycle in $A^{\prime}[1]$, because $\left(b^{\prime}\right)_{1}^{0}$ vanishes. As $\tilde{F}_{N, 1}^{N^{\prime}}$ induces an injection between the cohomology groups, and the image of $a^{\prime}$ under $\tilde{F}_{N, 1}^{N^{\prime}}$ is the coboundary $\left(b_{1}^{0} \circ \tilde{U}_{N}^{N^{\prime}}\right)(\omega)$, 
$a^{\prime}$ has to be itself a coboundary, but as $\left(b^{\prime}\right)_{1}^{0}$ is zero, this means that $a^{\prime}$ vanishes, so the same occurs to $\left(b_{1}^{0} \circ \tilde{U}_{N}^{N^{\prime}}\right)(\omega)$, for $\omega$ arbitrary. The lemma is proved.

Proof of Lemma 3.6 Before we begin the proof let us set some notation that will be useful. Let us write $\left.\tilde{f}\right|_{M}=\sum_{i \in \mathbb{N}_{0}} f^{i} \hbar^{i}$. Accordingly, if we denote by $\tilde{d}$ and $\bar{d}$ the differentials of $M_{\hbar}$ and $H^{\bullet}(M)_{\hbar}$, respectively, we have the expressions $\left.\tilde{d}\right|_{M}=$ $\sum_{i \in \mathbb{N}_{0}} d^{i} \hbar^{i}$ and $\left.\bar{d}\right|_{M}=\sum_{i \in \mathbb{N}_{0}} \bar{d}^{i} \hbar^{i}$, where we recall that $\bar{d}^{0}=0$. For an element $\tilde{m} \in M_{\hbar}$ of the form $\sum_{i \in \mathbb{N}_{0}} m_{i} \hbar^{i}$, and $N \in \mathbb{N}_{0}$, we shall denote by $\tilde{m}_{\leq N}$ the finite sum $\sum_{i=0}^{N} m_{i} \hbar^{i}$ in the quotient $M_{\hbar} \otimes_{k[\hbar]} k[\hbar] /\left(\hbar^{N+1}\right)$. Analogously, we shall write $\tilde{f}^{\leq N}$ the finite sum $\sum_{i=0}^{N} f^{i} \hbar^{i}$, which is a morphism of complexes from $H^{\bullet}(M)_{\hbar} \otimes_{k[\hbar]}$ $k[\hbar] /\left(\hbar^{N+1}\right)$ to $M_{\hbar} \otimes_{k[\hbar]} k[\hbar] /\left(\hbar^{N+1}\right)$, and the same for $\tilde{d} \leq N$ and $\bar{d} \leq N$, as differentials of $M_{\hbar} \otimes_{k[\hbar]} k[\hbar] /\left(\hbar^{N+1}\right)$ and $H^{\bullet}(M)_{\hbar} \otimes_{k[\hbar]} k[\hbar] /\left(\hbar^{N+1}\right)$, respectively. As before, we may denote the two latter deformations by $M_{\hbar^{(N+1)}}$ and $H^{\bullet}(M)_{\hbar^{(N+1)}}$, respectively.

In order to prove the lemma we shall prove that for $N \in \mathbb{N}$, the map $\tilde{f} \leq N$ induces a quasi-isomorphism between the corresponding bigraded deformations. We will proceed by induction. Note that it is true for $N=0$, by the construction of $f$. We shall now assume that the statement holds for $0, \ldots, N-1$, and we shall prove it for $N$, i.e. we shall show that $\tilde{f} \leq N$ induces a quasi-isomorphism from $H^{\bullet}(M)_{\hbar^{(N+1)}}$ to $M_{\hbar^{(N+1)}}$. It is easy to see that the statement implies the lemma.

Let us first prove that the cohomology map induced by $\tilde{f}^{\leq N}$ is injective. We have to prove that given $h=\sum_{j=0}^{N} h_{j} \hbar^{j} \in H^{\bullet}(M)_{\hbar^{(N+1)}}$ such that $\bar{d}^{\leq N}(h)=0$ and $\tilde{f}^{\leq N}(h)=\tilde{d}^{\leq N}(\alpha)$, for $\alpha=\sum_{j=0}^{N} \alpha_{j} \hbar^{j} \in M_{\hbar^{(N+1)}}$, then there exists $\beta=$ $\sum_{j=0}^{N-1} \beta_{j} \hbar^{j} \in H^{\bullet}(M)_{\hbar^{(N+1)}}$ such that $h=\bar{d}^{\leq N}(\beta)$. However, since $\bar{d}^{\leq N}(h)=0$ implies in particular that $\bar{d}^{\leq(N-1)}\left(h_{\leq(N-1)}\right)=0$, and $\tilde{f}^{\leq N}(h)=\tilde{d}^{\leq N}(\alpha)$ yields $\tilde{f}^{\leq(N-1)}\left(h_{\leq(N-1)}\right)=\tilde{d}^{\leq(N-1)}\left(\alpha_{\leq N-1}\right)$, by inductive hypothesis we have that there exists $\beta^{\prime}=\sum_{j=0}^{N-2} \beta_{j} \hbar^{j} \in H^{\bullet}(M)_{\hbar^{N}}$ such that $h_{\leq(N-1)}=\bar{d}^{\leq(N-1)}\left(\beta^{\prime}\right)$. By regarding $\beta^{\prime}$ in $H^{\bullet}(M)_{\hbar^{(N+1)}}$, we see that $h^{\prime}=h-\bar{d}^{\leq N}\left(\beta^{\prime}\right)=h_{N}^{\prime} \hbar^{N}$, for some $h_{N}^{\prime} \in H^{\bullet}(M)$, and

$$
\tilde{f}^{\leq N}\left(h^{\prime}\right)=\tilde{f}^{\leq N}\left(h-\bar{d}^{\leq N}\left(\beta^{\prime}\right)\right)=\tilde{d}^{\leq N}\left(\alpha-\tilde{f}^{\leq N}\left(\beta^{\prime}\right)\right) .
$$

Hence it suffices to prove our injectivity statement for $h^{\prime}=h_{N}^{\prime} \hbar^{N}$, or, equivalently, to assume that $h$ is of the form $h_{N} \hbar^{N}$, for $h_{N} \in H^{\bullet}(M)$. In this case, we have that $\tilde{d}^{\leq(N-1)}\left(\alpha_{\leq(N-1)}\right)$ vanishes, so by the surjectivity of the cohomology map induced by $\tilde{f}^{\leq(N-1)}$ we have that there exists $\beta=\sum_{j=0}^{N-1} \beta_{j} \hbar^{j} \in H^{\bullet}(M)_{\hbar^{N}}$ and $\gamma=\sum_{j=0}^{N-1} \gamma_{j} \hbar^{j} \in M_{\hbar^{N}}$ such that $\bar{d}^{\leq(N-1)}\left(\beta^{\prime}\right)=0$ and $\tilde{f}^{\leq(N-1)}(\beta)=$ $\alpha_{\leq(N-1)}+\tilde{d}^{\leq(N-1)}(\gamma)$. We shall now regard $\beta$ in $H^{\bullet}(M)_{\hbar^{(N+1)}}$ and $\gamma$ in $M_{\hbar^{(N+1)}}$. We claim that $\bar{d}^{\leq N}(\beta)=h$. The vanishing of $\bar{d}^{\leq(N-1)}\left(\beta^{\prime}\right)$ tells us that

$$
\bar{d}^{\leq N}(\beta)=\sum_{j=1}^{N} \bar{d}^{j}\left(\beta_{N-j}\right) \hbar^{N},
$$


so we must show that $h_{N}$ coincides with $\sum_{j=1}^{N} \bar{d}^{j}\left(\beta_{N-j}\right)$. Seeing that $f=f^{0}$ is injective, it suffices to prove that $f^{0}\left(h_{N}\right)$ and $f^{0}\left(\sum_{j=1}^{N} \bar{d}^{j}\left(\beta_{N-j}\right)\right)$ coincide. This follows from the following computations. We first consider

$$
\begin{aligned}
f^{0}\left(h_{N}\right) & =\sum_{i=0}^{N} d^{i}\left(\alpha_{N-i}\right)=d^{0}\left(\alpha_{N}\right)+\sum_{i=1}^{N} d^{i}\left(\alpha_{N-i}\right) \\
& =d^{0}\left(\alpha_{N}\right)+\sum_{i=1}^{N} d^{i}\left(\sum_{j=0}^{N-i} f^{j}\left(\beta_{N-i-j}\right)-\sum_{j=0}^{N-i} d^{j}\left(\gamma_{N-i-j}\right)\right) \\
& =d^{0}\left(\alpha_{N}\right)+\sum_{l=1}^{N} \sum_{i=1}^{l} d^{i} f^{l-i}\left(\beta_{N-l}\right)-\sum_{l=1}^{N} \sum_{i=1}^{l} d^{i} d^{l-i}\left(\gamma_{N-l}\right),
\end{aligned}
$$

where we have used $\tilde{f}^{\leq N}(h)=\tilde{d}^{\leq N}(\alpha)$ in the first equality, $\tilde{f}^{\leq(N-1)}(\beta)=\alpha_{\leq(N-1)}+$ $\tilde{d}^{\leq}(N-1)(\gamma)$ in the second equality, and we have only re-indexed the terms of the last two sums in the last member. Using that $\tilde{f}^{\leq N}$ is a morphism of complexes and $\tilde{d}^{\leq N}$ is a differential, the last member of the previous collection of equations coincides with

$$
\begin{aligned}
d^{0}\left(\alpha_{N}\right)- & \sum_{l=1}^{N} d^{0} f^{l}\left(\beta_{N-l}\right)+\sum_{l=1}^{N} \sum_{i=0}^{l-1} f^{i} \bar{d}^{l-i}\left(\beta_{N-l}\right)+\sum_{l=1}^{N} d^{0} d^{l}\left(\gamma_{N-l}\right) \\
= & f^{0}\left(\sum_{l=1}^{N} \bar{d}^{l}\left(\beta_{N-l}\right)\right)+d^{0}\left(\alpha_{N}-\sum_{l=1}^{N}\left(f^{l}\left(\beta_{N-l}\right)-d^{l}\left(\gamma_{N-l}\right)\right)\right) \\
& +\sum_{l=1}^{N-1} f^{l}\left(\sum_{j=1}^{N-l} \bar{d}^{j}\left(\beta_{N-j-l}\right)\right) \\
= & f^{0}\left(\sum_{l=1}^{N} \bar{d}^{l}\left(\beta_{N-l}\right)\right)+d^{0}\left(\alpha_{N}-\sum_{l=1}^{N}\left(f^{l}\left(\beta_{N-l}\right)-d^{l}\left(\gamma_{N-l}\right)\right)\right),
\end{aligned}
$$

where the first and last terms of the second member are obtained from splitting and re-indexing the third term of the first member, and we have used in the last equality that the last term of the second member vanishes, since, for each $l=1, \ldots, N-1$, $\sum_{j=1}^{N-l} \bar{d}^{j}\left(\beta_{N-j-l}\right)=0$, for this latter identity is tantamount to $\bar{d}^{\leq(N-1)}(\beta)=0$. We have that

$$
f^{0}\left(h_{N}-\sum_{l=1}^{N} \bar{d}^{l}\left(\beta_{N-l}\right)\right)=d^{0}\left(\alpha_{N}-\sum_{l=1}^{N}\left(f^{l}\left(\beta_{N-l}\right)-d^{l}\left(\gamma_{N-l}\right)\right)\right) .
$$

Taking into account that $f^{0}$ induces a quasi-isomorphism from $H^{\bullet}(M)$ to $M$, but the differential of $H^{\bullet}(M)$ is zero, we get that $f^{0}\left(h_{N}-\sum_{l=1}^{N} \bar{d}^{l}\left(\beta_{N-l}\right)\right)$ vanishes, which by the injectivity of $f^{0}$ implies that $h_{N}=\sum_{l=1}^{N} \bar{d}^{l}\left(\beta_{N-l}\right)$, which was to be shown. 
Let us now prove that the cohomology map induced by $\tilde{f} \leq N$ is surjective. Let $m=\sum_{j=0}^{N} m_{j} \hbar^{j} \in M_{\hbar^{(N+1)}}$ such that $\tilde{d}^{\leq N}(m)=0$. We want to show that there exist $h=\sum_{j=0}^{N} h_{j} \hbar^{j} \in H^{\bullet}(M)_{\hbar^{(N+1)}}$ and $n=\sum_{j=0}^{N} n_{j} \hbar^{j} \in M_{\hbar^{(N+1)}}$ such that $\bar{d}^{\leq N}(h)=0$ and $\tilde{f}^{\leq N}(h)=m+\tilde{d}^{\leq N}(n)$. Note that, from $\tilde{d}^{\leq N}(m)=0$ we have in particular that $\tilde{d}^{\leq(N-1)}\left(m_{\leq(N-1)}\right)=0$. Hence, by the inductive hypothesis there exist $h^{\prime}=\sum_{j=0}^{N-1} h_{j} \hbar^{j} \in H^{\bullet}(M)_{\hbar^{N}}$ and $n^{\prime}=\sum_{j=0}^{N-1} n_{j} \hbar^{j} \in M_{\hbar^{N}}$ such that $\bar{d} \leq(N-1)\left(h^{\prime}\right)=0$ and $\tilde{f} \leq(N-1)\left(h^{\prime}\right)=m_{\leq(N-1)}+\tilde{d} \leq(N-1)\left(n^{\prime}\right)$. We shall regard $h^{\prime}$ as an element of $H^{\bullet}(M)_{\hbar^{(N+1)}}$, and $n^{\prime}$ as an element in $M_{\hbar^{(N+1)}}$. We see that $\bar{d} \leq N\left(h^{\prime}\right)=\left(\sum_{j=1}^{N} \bar{d}^{j}\left(h_{N-j}\right)\right) \hbar^{N}$. We shall first show that it vanishes. In order to do so, we consider the identity $\tilde{f}^{\leq(N-1)}\left(h^{\prime}\right)=m_{\leq(N-1)}+\tilde{d}^{\leq(N-1)}\left(n^{\prime}\right)$, which can be equivalently rewritten as a collection of identities

$$
\sum_{i=0}^{j} f^{i}\left(h_{j-i}\right)=m_{j}+\sum_{i=0}^{j} d^{i}\left(n_{j-i}\right)
$$

for $j=0, \ldots, N-1$. We apply $d^{N-j}$ to the $j$-th of the preceding equations and we add up to get

$$
\sum_{j=0}^{N-1} \sum_{i=0}^{j} d^{N-j} f^{i}\left(h_{j-i}\right)=\sum_{j=0}^{N-1} d^{N-j}\left(m_{j}\right)+\sum_{j=0}^{N-1} \sum_{i=0}^{j} d^{N-j} d^{i}\left(n_{j-i}\right) .
$$

By re-indexing the sum in the first member and the last sum in the second one we get

$$
\sum_{l=1}^{N} \sum_{i=1}^{l} d^{i} f^{l-i}\left(h_{N-l}\right)=\sum_{j=0}^{N-1} d^{N-j}\left(m_{j}\right)+\sum_{l=1}^{N} \sum_{i=1}^{l} d^{i} d^{l-i}\left(n_{N-l}\right) .
$$

Using that $\tilde{f} \leq N$ is a morphism of complexes and $\tilde{d} \leq N$ is a differential, we rewrite the previous identity as

$$
-\sum_{l=1}^{N} d^{0} f^{l}\left(h_{N-l}\right)+\sum_{l=1}^{N} \sum_{i=0}^{l-1} f^{i} \bar{d}^{l-i}\left(h_{N-l}\right)=\sum_{j=0}^{N-1} d^{N-j}\left(m_{j}\right)-\sum_{l=1}^{N} d^{0} d^{l}\left(n_{N-l}\right) .
$$

By splitting and re-indexing the second summand of the first member, we obtain the equivalent identity

$$
\begin{aligned}
- & d^{0}\left(\sum_{l=1}^{N} f^{l}\left(h_{N-l}\right)\right)+f^{0}\left(\sum_{j=1}^{N} \bar{d}^{j}\left(h_{N-j}\right)\right)+\sum_{l=1}^{N-1} f^{l}\left(\sum_{j=1}^{N-l} \bar{d}^{j}\left(h_{N-j-l}\right)\right) \\
= & \sum_{j=0}^{N-1} d^{N-j}\left(m_{j}\right)-\sum_{l=1}^{N} d^{0} d^{l}\left(n_{N-l}\right) .
\end{aligned}
$$


Since, for each $l=1, \ldots, N-1, \sum_{j=1}^{N-l} \bar{d}^{j}\left(h_{N-j-l}\right)=0$, for this latter identity is tantamount to $\bar{d}^{\leq(N-1)}(h)=0$, we get that

$-d^{0}\left(\sum_{l=1}^{N} f^{l}\left(h_{N-l}\right)\right)+f^{0}\left(\sum_{j=1}^{N} \bar{d}^{j}\left(h_{N-j}\right)\right)=\sum_{j=0}^{N-1} d^{N-j}\left(m_{j}\right)-\sum_{l=1}^{N} d^{0} d^{l}\left(n_{N-l}\right)$.

Using that $\tilde{d}^{\leq N}(m)$ vanishes we conclude that

$$
f^{0}\left(\sum_{j=1}^{N} \bar{d}^{j}\left(h_{N-j}\right)\right)=d^{0}\left(\sum_{l=1}^{N} f^{l}\left(h_{N-l}\right)-m_{N}-\sum_{l=1}^{N} d^{l}\left(n_{N-l}\right)\right) .
$$

As $f^{0}$ induces a quasi-isomorphism from $H^{\bullet}(M)$ to $M$, but the differential of $H^{\bullet}(M)$ is zero, we get that $f^{0}\left(\sum_{j=1}^{N} \bar{d}^{j}\left(h_{N-j}\right)\right)$ vanishes, which by the injectivity of $f^{0}$ implies that $\sum_{j=1}^{N} \bar{d}^{j}\left(h_{N-j}\right)$ also vanishes, which was to be shown.

We will finally prove the surjectivity statement. We need to show that there exist $h_{N} \in H^{\bullet}(M)$ and $n_{N} \in M$ such that, for $h=h^{\prime}+h_{N} \hbar^{N}$ and $n=n^{\prime}+n_{N} \hbar^{N}$ elements in $H^{\bullet}(M)_{\hbar^{(N+1)}}$ and $M_{\hbar^{(N+1)}}$, respectively, $\bar{d}^{\leq N}(h)=0$ and $\tilde{f}^{\leq N}(h)=m+\tilde{d}^{\leq N}(n)$. In view of the fact that $\bar{d}^{0}$ is zero, we see that $\bar{d}^{\leq N}(h)=0$ holds no matter what value of $h_{N}$ we choose. We need only to deal with the second equation. Moreover, as $\tilde{f}^{\leq N-1)}\left(h^{\prime}\right)=m_{\leq(N-1)}+\tilde{d}^{\leq(N-1)}\left(n^{\prime}\right)$, the identity $\tilde{f}^{\leq N}(h)=m+\tilde{d}^{\leq N}(n)$ is tantamount to

$$
f^{0}\left(h_{N}\right)+\sum_{i=1}^{N} f^{i}\left(h_{N-i}\right)=m_{N}+\sum_{i=1}^{N} d^{i}\left(n_{N-i}\right)+d^{0}\left(n_{N}\right) .
$$

Hence, it suffices to prove that

$$
m_{N}+\sum_{i=1}^{N} d^{i}\left(n_{N-i}\right)-\sum_{i=1}^{N} f^{i}\left(h_{N-i}\right)
$$

belongs to the kernel of $d^{0}$, for in that case it can uniquely written as a sum of an element in the image of $f^{0}$ and a coboundary, i.e. elements $f^{0}\left(h_{N}\right)$ and $d^{0}\left(-n_{N}\right)$, which in our case shows the existence of the required $h_{N}$ and $n_{N}$. We shall thus compute

$$
\begin{aligned}
& d^{0}\left(m_{N}+\sum_{i=1}^{N} d^{i}\left(n_{N-i}\right)-\sum_{i=1}^{N} f^{i}\left(h_{N-i}\right)\right) \\
& =-\sum_{i=1}^{N} d^{i}\left(m_{N-i}\right)-\sum_{i=1}^{N} \sum_{j=1}^{i} d^{j} d^{i-j}\left(n_{N-i}\right)+\sum_{i=1}^{N} \sum_{j=1}^{i} d^{j} f^{i-j}\left(h_{N-i}\right)
\end{aligned}
$$




$$
\begin{aligned}
& -\sum_{i=1}^{N} \sum_{j=0}^{i-1} f^{j} \bar{d}^{i-j}\left(h_{N-i}\right) \\
= & -\sum_{i=1}^{N} d^{i}\left(m_{N-i}+\sum_{j=0}^{N-i} d^{j}\left(n_{N-i-j}\right)-\sum_{j=0}^{N-i} f^{j}\left(h_{N-i-j}\right)\right) \\
& -\sum_{j=0}^{N-1} f^{j}\left(\sum_{i=1}^{N-j} \bar{d}^{i}\left(h_{N-i-j}\right)\right),
\end{aligned}
$$

where we have used that $\tilde{d}^{\leq N}(m)=0, \tilde{d}^{\leq N} \circ \tilde{d}^{\leq N}=0$, and that $\tilde{f}^{\leq N}$ is a morphism of complexes in the first identity, and we have re-indexed the sums of the second member in the last identity. By the inductive hypothesis we have that $\tilde{f}^{\leq(N-1)}\left(h^{\prime}\right)=$ $m_{\leq(N-1)}+\tilde{d}^{\leq(N-1)}\left(n^{\prime}\right)$, which is tantamount to the vanishing of the arguments of $d^{i}$ (for $i=1, \ldots, N$ ) appearing in the first sum of the last chain of identities. Furthermore, the vanishing of $\tilde{d}^{\leq(N-1)}\left(h^{\prime}\right)$ is equivalent to the vanishing of the arguments of $f^{j}$ (for $j=0, \ldots, N-1$ ) appearing in the last sum of the previous chain of identities. This proves that $m_{N}+\sum_{i=1}^{N} d^{i}\left(n_{N-i}\right)-\sum_{i=1}^{N} f^{i}\left(h_{N-i}\right)$ belongs to the kernel of $d^{0}$, which in turn proves the surjectivity claim. The lemma is proved.

\section{References}

1. Fialowski, A., Penkava, M.: Deformation theory of infinity algebras. J. Algebra 255(1), 59-88 (2002)

2. Gerstenhaber, M.: On the deformation of rings and algebras. Ann. Math. 79(2), 59-103 (1964)

3. Gerstenhaber, M., Wilkerson, C. W.: On the deformation of rings and algebras. V. Deformation of differential graded algebras, Higher homotopy structures in topology and mathematical physics, Poughkeepsie, NY, : Contemp. Math., 227, Amer. Math. Soc. Providence, RI 1999, 89-101 (1996)

4. Herscovich, E.: Hochschild (co)homology and Koszul duality. http://arxiv.org/abs/1405.2247

5. Herscovich, E.: A higher homotopic extension of persistent (co)homology. http://arxiv.org/abs/1412. 1871

6. Heyneman, R.G., Radford, D.E.: Reflexivity and coalgebras of finite type. J. Algebra. 28, 215-246 (1974)

7. Kadeišvili, T. V.: On the theory of homology of fiber spaces, Russian. Int. Topol. Conference (Moscow State Univ., Moscow, 1979), Uspekhi Mat. Nauk. 35, 3(213), 183-188 (1980)

8. Kadeishvili, T. V.: The algebraic structure in the homology of an $A(\infty)$-algebra, Soobshch. Akad. Nauk Gruzin. SSR, 108(2), 249-252 (1983) (Russian, with English and Georgian summaries)

9. Kassel, C.: Quantum groups. Graduate texts in mathematics, pp. 155. Springer-Verlag, New York, xii+531 (1995)

10. Koszul, J.L.: Sur les opérateurs de dérivation dans un anneau, French. C. R. Acad. Sci. Paris 225, 217-219 (1947)

11. Koszul, J.L.: Sur 1'homologie des espaces homogènes, French. C. R. Acad. Sci. Paris 225, 477-479 (1947)

12. Lapin, S.V.: Differential perturbations and $D_{\infty}$-differential modules. Mat. Sb. 192(11), 55-76(Russian, with Russian summary); English transl., Sb. Math. 192(11-12), 1639-1659 (2001)

13. Lapin, S.V.: $D_{\infty}$-differential $A_{\infty}$-algebras and spectral sequences. Mat. Sb. 193(1), 119-142 (Russian, with Russian summary); English transl., Sb. Math. 193(1-2), 119-142 (2002)

14. Lapin, S. V.: $(D A)_{\infty}$-modules over $(D A)_{\infty}$-algebras, and spectral sequences. Izv. Ross. Akad. Nauk Ser. Mat. 66(3), 103-130 (Russian, with Russian summary); English transl., Izv. Math. 66 (3), 543-568 (2002) 
15. Lapin, S.V.: Multiplicative $A_{\infty}$-structure in the terms of spectral sequences of fibrations, Russian, with English and Russian summaries, Fundam. Prikl. Mat., 14, 2008, 6, 141-175, J. Math. Sci. (N. Y.), 164(1), 95-118 (2010)

16. Lefèvre-Hasegawa, K.: Sur les $A_{\infty}$-catégories, French, Thesis (Ph.D.)-Université Paris 7, Paris, France, 2003. http://arxiv.org/abs/math/0310337. Corrections at http://www.math.jussieu.fr/ keller/ lefevre/TheseFinale/corrainf.pdf

17. Leray, J.: Structure de l'anneau d'homologie d'une représentation, French. C. R. Acad. Sci. Paris 222, 1419-1422 (1946)

18. Lu, D.M., Palmieri, J.H., Wu, Q.S., Zhang, J.J.: $A_{\infty}$-algebras for ring theorists. In: Proceedings of the International Conference on Algebra, pp. 91-128 (2004)

19. Lu, D.-M., Palmieri, J.H., Wu, Q.-S., Zhang, J.J.: A-infinity structure on Ext-algebras. J. Pure Appl. Algebra 213(11), 2017-2037 (2009)

20. Massey, W.S.: Exact couples in algebraic topology. I, II, Ann. Math. 56(2), 363-396 (1952)

21. Massey, W.S.: Exact couples in algebraic topology. III, IV, V, Ann. Math. 57(2), 248-286 (1953)

22. Massey, W.S.: Products in exact couples. Ann. Math. 59(2), 558-569 (1954)

23. McCleary, J.: A history of spectral sequences: origins to: History of topology. North-Holland, Amsterdam. 1999, 631-663 (1953)

24. McCleary, J.: A user's guide to spectral sequences. Cambridge Studies in Advanced Mathematics, pp. 58, 2. Cambridge University Press, Cambridge, xvi+561 (2001)

25. Prouté, A.: $A_{\infty}$-structures. Modèles minimaux de Baues-Lemaire et Kadeishvili et homologie des fibrations, French. Reprint of the: original; With a preface to the reprint by Jean-Louis Loday. Repr. Theory Appl. Categ. 21(2011), 1-99 (1986)

26. Sagave, S.: DG-algebras and derived $A_{\infty}$-algebras. J. Reine Angew. Math. 639, 73-105 (2010)

27. Stasheff, J.D.: Homotopy associativity of $H$-spaces. I, II, Trans. Amer. Math. Soc. 108 (1963), 275292; ibid., 108, 293-312 (1963)

28. Weibel, C. A.: An introduction to homological algebra. Cambridge Studies in Advanced Mathematics, pp. 38. Cambridge University Press, Cambridge, xiv+450 (1994)

29. Wu, E.: Deformation and Hochschild cohomology of $A_{\infty}$-algebras, Shanghai, China. Thesis (M.Sc.)Zhejiang University (2002). http://www.math.uwo.ca/ ewu22/deformationofA-infinity.dvi 